The Astrophysical Journal Supplement Series, 35: 221-237, 1977 October

(C) 1977. The American Astronomical Society. All rights reserved. Printed in U.S.A.

\title{
THE PHASE DIAGRAM AND TRANSPORT PROPERTIES FOR HYDROGEN-HELIUM FLUID PLANETS
}

\author{
D. J. STEVEnson AND E. E. SAlPETER \\ Center for Radiophysics and Space Research and Physics Department, Cornell University \\ Received 1976 June 23; accepted 1977 April 13
}

\begin{abstract}
Hydrogen and helium are the major constituents of Jupiter and Saturn, and phase transitions can have important effects on the planetary structure. In this paper, the relevant phase diagrams and microscopic transport properties are analyzed in detail. The following paper (Paper II) applies these results to the evolution and present dynamic structure of the Jovian planets.

Pure hydrogen is first discussed, especially the nature of the molecular-metallic transition and the melting curves for the two phases. It is concluded that at the temperatures and pressures of interest $\left(T \approx 10^{4} \mathrm{~K}, P \approx 1-10 \mathrm{Mbar}\right.$ ), both phases are fluid, but the transition between them might nevertheless be first-order. The insulator-metal transition in helium occurs at a much higher pressure ( $\sim 70$ Mbars) and is not of interest.

The phase diagrams for both molecular and metallic hydrogen-helium mixtures are discussed. In the metallic mixture, calculations indicate a miscibility gap for $T \leqslant 10^{4} \mathrm{~K}$. Immiscibility in the molecular mixture is more difficult to predict but almost certainly occurs at much lower temperatures. A fluid-state model is constructed which predicts the likely topology of the threedimensional phase diagram. The greater solubility of helium in the molecular phase leads to the prediction that the $\mathrm{He} / \mathrm{H}$ mass ratio is typically twice as large in the molecular phase as in the coexisting metallic phase. Under these circumstances a "density inversion" is possible in which the molecular phase becomes more dense than the metallic phase.

The partitioning of minor constituents is also considered: The deuterium/hydrogen mass ratio is essentially the same for all coexisting hydrogen-helium phases, at least for $T \geqslant 5000 \mathrm{~K}$. The partitioning of $\mathrm{H}_{2} \mathrm{O}, \mathrm{CH}_{4}$, and $\mathrm{NH}_{3}$ probably favors the molecular (or helium-rich) phase. Substances with high conduction electron density (e.g., Al) may partition into the metallic phase.

Electronic and thermal conductivities, viscosity, helium diffusivity, and Soret coefficient are evaluated for the fluid molecular and metallic phases, all to at least order-of-magnitude accuracy. The properties of the metallic phase are typical of a liquid alkali metal, and those of the molecular phase are typical of a dense neutral fluid (except that the conductivities may be almost metallic at the transition pressure). The opacities of molecular hydrogen and solar-composition mixtures are discussed for $T \approx 500 \mathrm{~K}$, where molecular hydrogen alone may be insufficiently opaque to ensure convection in the Jovian planets. Sufficient opacity to initiate convection is probably supplied by the minor constituents. Current uncertainties are assessed.
\end{abstract}

Subject headings: equation of state - planets: interiors

\section{INTRODUCTION}

Hydrogen and helium comprise roughly $85 \%$ of the total planetary mass in our solar system, and are the major constituents of Jupiter and Saturn. They are also the simplest atomic species, so their thermodynamic and transport properties should be amenable to first-principles calculation at those pressures which are presently unattainable by experiment.

There has been recent intensive modeling of the interior of Jupiter by several groups (Podolak and Cameron 1975; Zharkov et al. 1975; Hubbard and Slattery 1976; Stevenson and Salpeter 1976; Podolak 1977), and much attention has been given to the equation of state and other thermodynamic derivatives for hydrogen and hydrogen-helium mixtures. However, all these models assume a homogeneous mixture of hydrogen and helium. This assumption may be fundamentally incompatible with the phase diagram of hydrogen-helium mixtures.

The present paper and the following paper (Stevenson and Salpeter 1977, hereafter Paper II) consider in detail the phase diagram for hydrogen-helium mixtures, and its implications for the interiors of the Jovian planets. Since these implications depend on details of the transport (including fluid-dynamical) processes, the present paper also contains a survey of the current knowledge of the microscopic transport properties of dense hydrogen-helium mixtures.

The present paper concentrates on the condensedmatter physics of such mixtures, with emphasis given to the pressure-temperature domain appropriate to Jupiter and Saturn. The emphasis is on the fluid state, which is almost certainly applicable to the 
present interiors of Jupiter and Saturn, but there is also a discussion of melting curves for the hydrogenhelium phases. Since the Jovian planets contain constituents other than hydrogen and helium, the effects of these are considered briefly. The equation of state and other thermodynamic derivatives are not discussed in detail here, but an extensive review is to be found elsewhere (Stevenson and Salpeter 1976).

In $\S$ II, we discuss the properties of pure hydrogen and helium, especially the melting curves and insulator-metal transitions. The nature of the molecularmetallic hydrogen phase transition is not yet well understood, but is expected to occur at $2 \mathrm{Mbar} \leqslant P \leqslant$ $4 \mathrm{Mbar}$ and to be first-order at least until $T \approx 10^{3} \mathrm{~K}$ and quite possibly even for $T \geqslant 10^{4} \mathrm{~K}$. At $10^{4} \mathrm{~K}$, the two phases are certainly both fluid. The insulatormetal transition in helium occurs at $P \approx 70 \mathrm{Mbar}$, which is too high to be of interest for the Jovian planets.

In $\S$ III, calculations (Stevenson 1975) for the phase diagram of metallic hydrogen-helium mixtures are reviewed. A miscibility gap is predicted for a solar composition mixture at megabar pressures and temperatures less than $10^{4} \mathrm{~K}$.

In $\S \mathrm{IV}$, the phase diagram of molecular hydrogenhelium mixtures is discussed. Unlike the metallic phase, where an essentially first-principles calculation can be made, calculations for the molecular phase must rely on semiempirical intermolecular potentials, and are necessarily suspect. However, the prediction that helium is more soluble in molecular hydrogen than in metallic hydrogen is reliable.

In $\S \mathrm{V}$, the conclusions of the previous sections are used to model a total phase diagram which simultaneously accounts for the first-order character of the molecular-metallic hydrogen transition, the limited solubility of helium, and the thermodynamic preference for helium to be dissolved in the molecular hydrogen rather than metallic hydrogen phase. This model may be numerically imprecise, but is expected to predict the correct topology of the (three-dimensional) phase diagram. The predicted phase diagrams are similar to those suggested by Smoluchowski (1973). This model contains two other useful features: First, it predicts the circumstances for which a "density inversion" occurs (i.e., when a helium-poor metallic phase is less dense than a coexisting heliumrich molecular phase). Second, it predicts the limited range of metastability for the molecular phase in the metallic region, and vice versa.

In $\S$ VI, minor constituents are discussed. Immiscibilities appear unlikely, but the partitioning of minor constituents among the various hydrogen-helium phases is undoubtedly nonuniform. A special case is deuterium, for which calculations indicate that the deuterium/hydrogen mass ratio in each phase is essentially uniform, at least for $T \geqslant 5000 \mathrm{~K}$. A model is proposed for other minor constituents, in which partitioning is in favor of the phase with the most similar electron density at the Wigner-Seitz cell boundary. This model predicts that $\mathrm{H}_{2} \mathrm{O}, \mathrm{NH}_{3}$, and $\mathrm{CH}_{4}$ prefer molecular or helium-rich phases, but the degree of nonuniform partitioning is probably less than an order of magnitude.

Section VII is a summary of the microscopic transport properties of the metallic phase. Electronic and thermal conductivities, viscosity, and helium diffusivity are given particular attention.

In $\S$ VIII, the corresponding transport properties of the molecular phase are considered. In addition, the opacities of dense molecular hydrogen and solarcomposition mixtures are discussed, especially for temperatures of order $500 \mathrm{~K}$.

Section IX concludes with an assessment of current uncertainties. In the following paper (Paper II), specific thermal and compositional evolutions of a hydrogen-helium planet like Jupiter are discussed semiquantitatively.

\section{THE PURE PHASES}

\section{a) Hydrogen}

Even at $T=0 \mathrm{~K}$, there must be some sufficiently high density for which the Pauli exclusion principle precludes the existence of molecules or localized states and dense hydrogen becomes a Coulomb plasma: protons immersed in an almost uniform, degenerate sea of electrons. Wigner and Huntington (1935) pointed out that this atomic state would be analogous to the conventional alkali metals and therefore metallic. This atomic state is referred to as "metallic hydrogen" to indicate that its high conductivity is a consequence of itinerant electronic states in a monovalent metal, rather than being a consequence of temperature.

If the density is reduced sufficiently and the temperature is low enough, then it becomes thermodynamically favorable to pair the protons in the form of $\mathrm{H}_{2}$ molecules. This is the experimentally accessible molecular phase. The transition between the molecular and metallic phases occurs at a pressure given approximately by the dissociation energy per molecule divided by the volume per molecule: a few megabars. The molecular phase exists in both solid and liquid forms, and the metallic phase is expected to behave likewise. Additional low-temperature phases that cannot be categorized as either metallic or molecular are not yet rigorously excluded, but neither are they indicated experimentally or theoretically. We discuss below the metallic phase, the molecular phase, and the metallic-molecular transition.

\section{i) Metallic Hydrogen}

The evaluation of the thermodynamics of the alkali metals from first principles is well established for both the solid and fluid phases (see, for example, Stroud and Ashcroft 1972), and the properties of metallic hydrogen can be evaluated in a similar fashion. There are two important respects in which metallic hydrogen is unlike the conventional alkalis: the effective electronion interaction is stronger (because there are no core states) and quantum effects for the ions (i.e., protons) are significant (because of the larger electron-ion mass ratio). The former is particularly important at low densities whereas the latter is most important at 
high densities and low temperatures. Hubbard and Smoluchowski (1973) have an excellent review of earlier work on metallic hydrogen and we comment here on more recent work, with a particular emphasis on the solid-fluid transition.

The most recent calculations for a static metallic hydrogen lattice by a variety of perturbative and nonperturbative techniques are in excellent agreement (Ross and McMahan 1976). The most favored lattice structure has not been established, but this is unimportant for most purposes since the energy difference between structures is so small. It has been suggested that the lowest energy structure is highly anisotropic (Brovman, Kagan, and Kholas 1972), but this conclusion is premature (Hammerberg and Ashcroft 1974; Ross and McMahan 1976). The finite temperature and zero-point motion corrections are not as well understood (Brovman, Kagan, and Kholas 1972; Caron 1974; Straus and Ashcroft 1977) but appear to be describable by a Debye model in which two Debye temperatures are defined-one for the longitudinal modes and one for the transverse modes. Most of these calculations indicate that the transverse modes are "soft," and in some instances the stability of the lattice is in doubt.

Recent fluid-state calculations have been made by Hubbard and Slattery (1971), Stevenson (1975), Hansen and Vieillefosse (1976), and Hubbard and DeWitt (1976). As with all simple metals, the thermodynamic derivatives with respect to volume or pressure (e.g., the equation of state) are very similar to the solid. Thermodynamic derivatives with respect to temperature (e.g., entropy) are, of course, substantially different from the solid, but the various methods used are substantially in agreement. The results are summarized in Stevenson and Salpeter (1976).

The only rigorous way to calculate the melting temperature of a substance (assuming, of course, that the solid state exists) is by equating the Gibbs free energies for the two phases. This is a very difficult procedure since, although the energy of each phase is very accurately known, most of the energy is structureindependent, and the energy difference between the phases is very small at all temperatures. Pollock and Hansen (1973) used their Monte Carlo results for each phase to deduce a melting temperature $T_{M}$ for metallic hydrogen and found

$$
T_{M} \approx 1500 \rho^{1 / 3} \mathrm{~K}
$$

by equating Gibbs energies, where $\rho$ is the density in $\mathrm{g} \mathrm{cm}^{-3}$. This is probably an upper bound since it does not include the effects of screening on the ion-ion interaction. A similar calculation, including screening, has been attempted by Stevenson and Straus (unpublished) using the solid-state free energies of Straus, Ashcroft, and Beck (1977) and the fluid-state free energies of Stevenson (1975). The fluid state appeared to always have lower energy, but the energy difference was found to be comparable to the errors inherent in the calculations. The conclusion reached is that equation (1) is indeed an upper bound.
Several other methods have been tried for estimating $T_{M}$. One common method is Lindemann's rule, but this method is unreliable for a substance such as metallic hydrogen, where $T_{M}$ is less than the Debye temperature (Stevenson and Ashcroft 1974). Another method is based on the solidification of the classical hard sphere liquid at $45 \%$ packing (Wainwright and Alder 1958), but this method predicts $T_{M} \approx 1100 \mathrm{~K}$ at $\rho=1 \mathrm{~g} \mathrm{~cm}^{-3}$, a value that may be too low for the classical theory to be applicable (Stevenson 1975).

At sufficiently high densities, where screening is unimportant, the large zero-point motion of the protons precludes a solid at $T=0 \mathrm{~K}$. The density above which there is no solid is about $10^{4}-10^{5} \mathrm{~g} \mathrm{~cm}^{-3}$ (Glyde et al. 1976; Van Horn 1967). This is too high to be of interest in the giant planets. Whether screening precludes a solid phase at much lower densities has not yet been established.

If the solid exists at $\rho \approx 1 \mathrm{~g} \mathrm{~cm}^{-3}$, then it is most likely a superconductor below about $100 \mathrm{~K}$ (Ashcroft 1968; Caron 1974). If no solid exists, then an anisotropic superfluid may be possible. However, these lowtemperature effects are not relevant to the giant planets where $T \geqslant 10^{4} \mathrm{~K}$ is implied (see Paper II), and the fluid state is ensured without invoking quantum effects. Subsequent discussion of the metallic state in this paper is mainly for the fluid.

\section{ii) Molecular Hydrogen}

At $P \leqslant 0.1 \mathrm{Mbar}$ this phase is quite well understood experimentally, but the experimental uncertainty increases as the pressure increases (Ross 1974). Past theoretical calculations are no more accurate than experiment at the highest pressures because of the failure of the pair potential approximation (Ree and Bender 1974), but recent band structure calculations (Ramaker, Kumar, and Harris 1975; Friedli and Ashcroft 1976) are potentially capable of greater accuracy. Nevertheless, it is still necessary for most purposes to resort to semiempirical pair potentials that are compatible with the experimental shock data (Ross 1974) yet are also plausible modifications of first-principles calculations (McMahan, Beck, and Krumhansl 1974). The most recent first-principles calculations of the effective pair potential are by Etters, Danilowicz, and England (1975) and include detailed consideration of the anisotropy of the interaction. They found that the energy associated with molecular orientation becomes larger than the zeropoint energy as the pressure increases, so that the molecules become "frozen" into a particular configuration at $T=0 \mathrm{~K}$ and $P \geqslant 0.3 \mathrm{Mbar}$. The preferred lattice configuration appears to be the tetragonal $\gamma$-nitrogen structure rather than the essentially cubic $\alpha$-nitrogen structure. At megabar pressures, the energy required to rotate a molecule is equivalent to a temperature of order $2000 \mathrm{~K}$.

The excited states of molecular hydrogen are even less well understood than the ground state. The characteristic temperature for intramolecular vibration appears to be only weakly dependent on density and may actually decrease at the highest pressures (Silver 
and Stevens 1973). Electronic excitation and molecular dissociation at the highest pressures are not understood quantitatively, but are expected to be important. The thermodynamic uncertainties are discussed in Stevenson and Salpeter (1976).

Recent fluid-state calculations have been made by Ross (1974) and Stevenson and Salpeter (1976), assuming a sphericalized potential. As usual, the solid and fluid equations of state at high pressure are very similar, provided the same potential is used for each. These fluid-state calculations suggest a melting temperature $T_{M}$, according to the criterion that the packing fraction in the equivalent hard sphere liquid not exceed $45 \%$ (Wainwright and Alder 1958). For $\rho \geqslant 0.4 \mathrm{~g} \mathrm{~cm}^{-3}$, Stevenson (1976a) finds

$$
T_{M} \approx 2800 \rho^{2} \mathrm{~K},
$$

and Ross (1974) has obtained similar results. This result is uncertain by perhaps $50 \%$, because of the uncertainty in the effective potential, and also assumes that the potential can be approximated by a spherical average. This may be valid for the fluid phase, but if the solid has an ordered configuration of molecular orientations, then the hard sphere criterion may be invalid. However, similar values for $T_{M}$ are suggested by the Lindemann criterion (Neece, Rogers, and Hoover 1971).

In summary, the thermodynamics of molecular hydrogen at $P \geqslant 0.1 \mathrm{Mbar}$ are not well understood, and the best constraint on the equation of state is the experimental shock data. The melting temperature is known to about a factor of 2 , but is nevertheless almost certainly too low for the solid phase to exist in the present giant planets (see Paper II). Unlike metallic hydrogen, the molecular phase is increasingly classical as the pressure increases (Krumhansl and Wu 1968). Despite the uncertainties, we shall find that useful quantitative calculations can be made.

\section{iii) The Molecular-metallic Transition}

There has not yet been a convincing experimental verification of this transition, although two claims (Grigoryev et al. 1972; Vereschchagin, Yakovlev, and Timofeev 1975a) have been made. The transition pressure is therefore estimated by theoretical calculations for the energies of the two phases and the usual common tangent construction. The most recent and most accurate calculations for $T=0 \mathrm{~K}$ (Ross 1974) predict a transition pressure of between 2 and 4 Megabars. The factor of 2 uncertainty reflects the uncertainty in the molecular equation of state. It has been suggested that there is a comparable uncertainty arising from the possibly incorrect usage of the free electron correlation energy in the metallic-state calculation (Monkhurst and Oddershede 1973; Ross and McMahan 1976). Since the correlation energy is very weakly density-dependent, this would represent an uncertainty in the energy scale and not in the equation of state. (Computation of the correlation energy in the molecular state from first principles would be even more difficult. This problem does not arise in most calculations at present, which rely on the experimental properties of molecular hydrogen.) In conclusion, it seems almost certain that the transition pressure exceeds 1 Mbar. An upper limit cannot be established with the same certainty, but is probably about $5 \mathrm{Mbar}$. For the "most likely" transition pressure of $\sim 3$ Mbar, the densities at transition are roughly $0.9 \mathrm{~g}$ $\mathrm{cm}^{-3}$ for the molecular phase and $1.1 \mathrm{~g} \mathrm{~cm}^{-3}$ for the metallic phase.

It is likely that the transition is first-order at zero temperature because of the apparent dissimilarity of the two phases (for example, the large predicted density change at the transition). The nature of the transition is directly related to the sign of the microscopic "surface energy" between the phases. In a simple model to be described below, this sign is found to be positive.

As the temperature increases, entropy considerations ensure some "mixing" of the phases, and some temperature must exist beyond which the transition ceases to be first-order. It is possible that the upper limit of the first-order character is coincident with the melting curve; i.e., there exists a triple point at which metallic solid, molecular solid, and a "mixed" fluid phase are in mutual equilibrium (cf. Trubitsyn 1972). On the other hand, Landau and Zel'dovich (1943) favor at least one critical point in the fluid region, in which case distinct metallic fluid and molecular fluid phases could coexist. The solid-fluid transition is a rather subtle one, from an energetic standpoint, with the main change being the absence of long-range order in the fluid phase. Indeed, the volume change upon melting for either phase is very small (less than $3 \%$ ), whereas the volume change that accompanies the molecular-metallic transition is comparatively large $(20-30 \%)$. In other words, the electronic structures of the fluid and the solid are very similar whether one considers the molecular or the metallic state; but the electronic structure for molecular hydrogen differs substantially from that for metallic hydrogen.

Nevertheless, two calculations (Kerley 1972; Aviram et al. 1976) suggest that the transition is continuous in the fluid state. Neither calculation can be regarded as satisfactory, since neither treats the two extremes (pure molecular and pure metallic) with a comparable degree of sophistication. Calculation of the phase diagram requires a very careful calculation of the Gibbs energy for an arbitrary mixture of the two phases. We shall not attempt this, but the relevant energies in such a calculation may be indicated by the following model.

We first note that it is not meaningful to think of the electrons as being "localized" in very dense molecular hydrogen. With the exception of small regions centered on each proton (in which the electron density is highly nonuniform in both molecular and metallic phases), the electron density is quite uniform. In the language of band theory, dense molecular hydrogen is insulating because it is divalent, with a nonvanishing indirect band gap. In fact, this band gap is much less than the band width at megabar pressures (Friedli and Ashcroft 1976). 
Our model rests on three hypotheses:

1. A hydrogen molecule exists as a bound, metastable state when surrounded by metallic hydrogen at $P=P_{t}$, the transition pressure. This hypothesis is crucial to the model, but difficult to verify.

2. The volume per electron in a mixture of the metallic and molecular phases is approximately independent of position, i.e., the electron density does not fluctuate greatly according to whether one is near a molecule or near an unbound proton. This is reasonable, since the Thomas-Fermi screening length is comparable to typical interproton distances.

3 . The energy of a neutral entity (i.e., a "molecule," or an unbound proton together with a screening cloud of one electronic charge) is a function only of the volume it occupies. This is the Wigner-Seitz hypothesis, and is expected to be quite accurate.

Figure 1 shows the $T=0 \mathrm{~K}$ internal energies of the two pure phases (Ross 1974). Consider the formation of a molecule in the metallic state at the transition pressure $P_{t} \approx 3$ Mbar. According to hypothesis 2, this occurs with essentially no volume change. According to hypothesis 3 , the cost in energy per proton is just the difference $\Delta E_{1}$ shown in Figure 1. Similarly, $\Delta E_{2}$ is the energy cost per proton for breaking up a molecule in the molecular phase. Since these energies are both positive, we have established from very simple considerations that the microscopic surface energy, between the two phases, is positive. The transition will be first-order until a temperature $T_{c}$ such that the entropy of mixing, roughly $k_{B} T_{c} \ln 2$ (where $k_{B}$ is Boltzmann's constant), is comparable to $\Delta E_{1}$ or $\Delta E_{2}$. This predicts that $T_{c}$ is a few thousand kelvins.

This model has been quantified (Stevenson 1976a) by expressing the Gibbs free energy per proton as a function $G(x, P)$ of pressure $P$ and of the fraction $x$ of the protons which are bound in molecules. The transition pressure, critical temperature, and critical

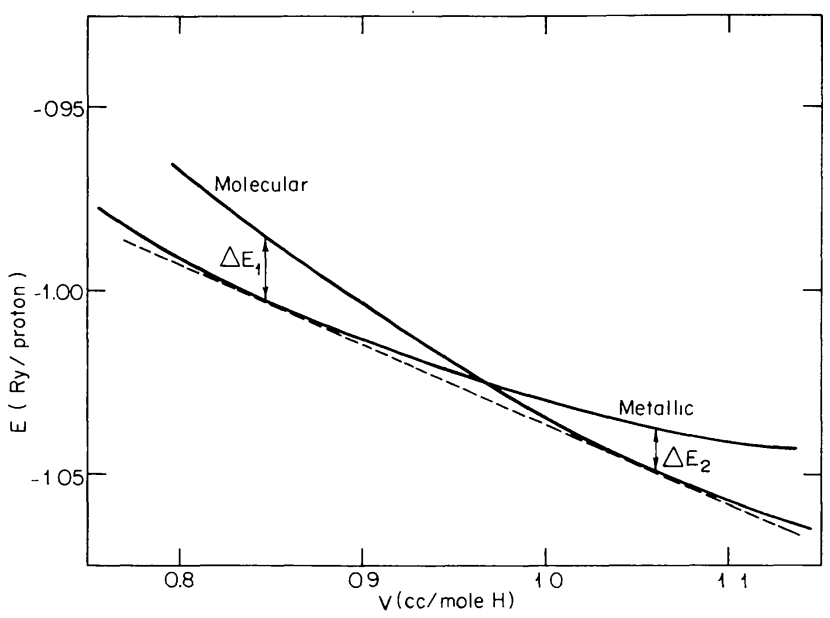

FIG. 1.-Internal energy at $T=0 \mathrm{~K}$ for molecular and metallic phases. Dashed line is a common tangent with slope $P=3$ Mbar. See text for discussion of $\Delta E_{1}, \Delta E_{2}$. concentration are found from simultaneous solution of the equations

$$
\frac{\partial G}{\partial x}=\frac{\partial^{2} G}{\partial x^{2}}=\frac{\partial^{3} G}{\partial x^{3}}=0,
$$

where the derivatives are at constant pressure and temperature. The results are $P_{t} \approx 3 \mathrm{Mbar}, T_{c} \approx$ $3500 \mathrm{~K}$, and $x_{c} \approx 0.4$.

The significance of this model is not in the numerical results, but rather in the identification of the relevant energies. According to this model, the relevant energy characterizing the transition is an order of magnitude smaller than the dissociation energy of an isolated
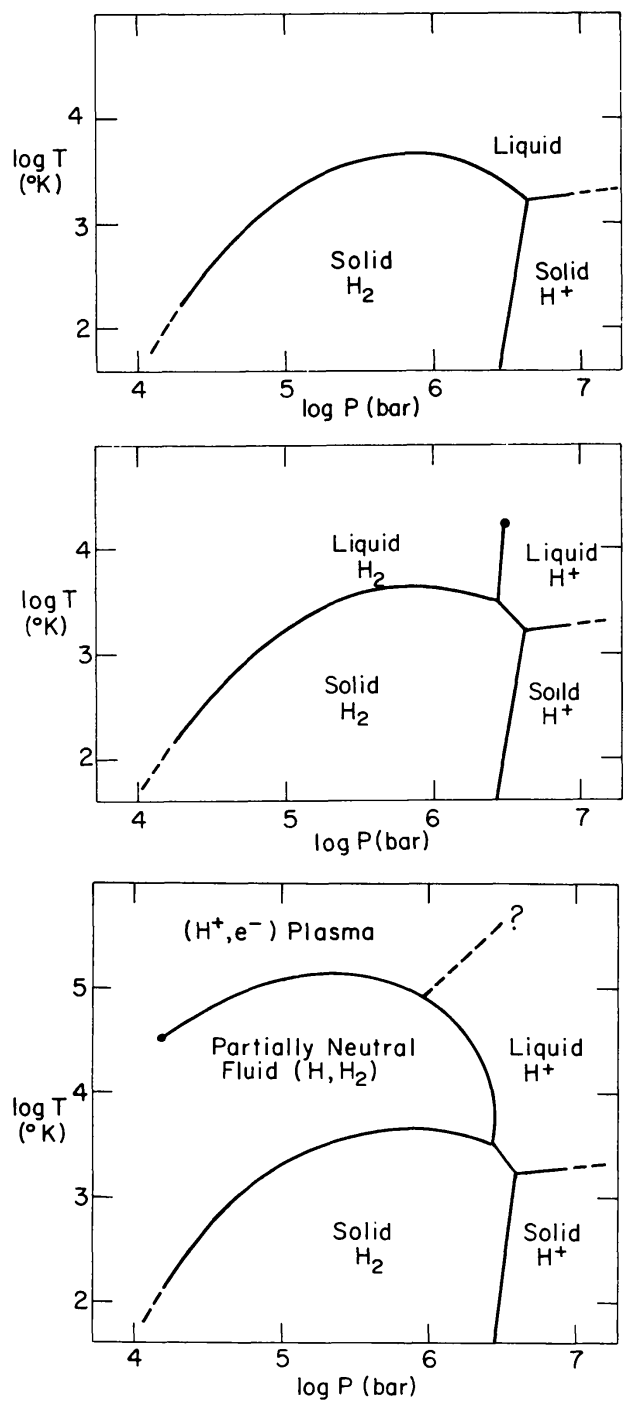

Fig. 2.-Several possible phase diagrams of high-pressure hydrogen. In (a) (top) no critical point exists. In (b) (middle) there is a critical point so that two distinct liquid states coexist. In (c) (bottom) the low-temperature phase diagram of $(b)$ is joined in a natural way to the high-temperature phase diagram of Filinov and Norman (1975). The high-temperature dashed line represents the onset of degeneracy or even the possibility of another first-order transition (cf. Landau and Zel'dovich 1943). In all these phase diagrams, the solid metallic phase is assumed to exist. 
hydrogen molecule. The estimated critical temperature is comparable to the melting temperature of the molecular phase at $\rho \approx 1 \mathrm{~g} \mathrm{~cm}^{-3}$, but this is purely coincidental. Our model may, however, be misleading and our first hypothesis may not even hold. An upper limit to $T_{c}$ is of order $10^{5} \mathrm{~K}$, and any value in the range $10^{3} \leqslant T_{c} \leqslant 10^{5} \mathrm{~K}$ cannot presently be discounted. In Figure 2, three possible high-pressure phase diagrams of hydrogen are shown to illustrate the large uncertainty. The bottom phase diagram in Figure 2 is highly unconventional, but is a natural extension of a recent suggestion by Filinov and Norman (1975) that hydrogen undergoes a gas-liquid transition, analogous to that of cesium, in which the gas is almost fully ionized nondegenerate atomic hydrogen, and the "liquid" is partially ionized atomic hydrogen. This last phase diagram is also in the spirit of the Landau-Zel'dovich (1943) hypothesis.

To conclude, there is a quite high probability that the molecular-metallic transition is first-order in part of the fluid phase. The transition is possibly firstorder even at $10,000 \mathrm{~K}$, the relevant temperature for the present interior of Jupiter (see Paper II).

\section{b) Helium}

Helium is the most difficult element to ionize and the most difficult substance to metallize. Estimates of the insulator-metal transition pressure range from 20 Mbar to 100 Mbar (Simcox and March 1962; Trubitsyn 1967; Brust 1972; Ross 1972; Østgaard 1974; Stevenson 1976a), but the most reliable of these estimates are near the upper limit. Since this transition is so far removed from the hydrogen transition, we will effectively ignore it, but it may be important in cold stars of low mass.

There are two approaches to the thermodynamics of helium. At low pressures, an interatomic pair potential compatible with experiment can be used (Trubitsyn 1967). At sufficiently high pressures $(P \geqslant$ 10 Mbars), a first-principles approach analogous to metallic hydrogen can be used. This approach is accurate provided the band gap (between valence and conduction bands) is less than the valence band width, and does not require that the helium actually be metallic. The overlap between the two procedures is substantial and readily leads to a smooth interpolation between the low-pressure and high-pressure limits (Trubitsyn 1967). The considerations in the next three sections are not sensitive to the slight mismatch of the two approaches.

The melting temperature can be estimated from the criterion for freezing of a hard sphere fluid or from Lindemann's rule. At low pressures, the hard sphere criterion predicts $T_{M} \approx 1700 \mathrm{~K}$ at $P=1 \mathrm{Mbar}$ and $T_{M} \approx 4500 \mathrm{~K}$ at $P=4 \mathrm{Mbar}$ (Stevenson 1976a). At high pressures, the melting temperature increases less rapidly with

$$
T_{M} \approx 4700 \rho^{1 / 3} \mathrm{~K}
$$

for $\rho$ in $\mathrm{g} \mathrm{cm}^{-3}$ (Trubitsyn 1967; Stevenson and Ashcroft 1974). For example, $T_{M} \approx 10,000 \mathrm{~K}$ at $P=50$ Mbar. Like hydrogen, helium also melts at $T=0 \mathrm{~K}$ for a sufficiently high density (Stevenson and Ashcroft 1974), but this is of no interest for the giant planets.

\section{METALLIC HYDROGEN-HELIUM MIXTURES}

We first consider fluid mixtures. The existence of miscibility gaps in many liquid metal mixtures is well known experimentally, but is difficult to predict theoretically since it depends on subtle free energy differences between the mixed and separated states. Nevertheless, it has recently become possible to predict phase diagrams to roughly $10 \%$ accuracy, at least for simple metals where the interactions are well known (Stroud 1973). These calculations are based on a nearly free electron theory of metals, and a hard sphere perturbation theory for the structural properties of the liquid.

Metallic hydrogen-helium mixtures differ from alloys currently accessible in the laboratory, in that there are no "core" electrons to contend with, so the accuracy of a calculation is limited only by our knowledge of the dielectric response of the electron gas and the structural properties of the liquid. On the other hand, the "bare" protons and $\alpha$-particles are rather severe perturbations on the electron gas, so it is desirable to evaluate the electronic response to higher order than the usual low-order (linear response) approximation. A recent calculation (Stevenson 1975) evaluates the Gibbs energy to third-order in the electron-ion interaction, and uses a perturbation theory of fluids. This calculation predicts a miscibility gap, the pressure dependence of which is shown in Figure 3. Below the critical line, a mixture containing roughly $40 \%$ helium by number will phase-separate into helium-rich and hydrogen-rich phases. Below the dashed line, any mixture with a composition between $10 \%$ and $70 \%$ helium will similarly phase-separate.

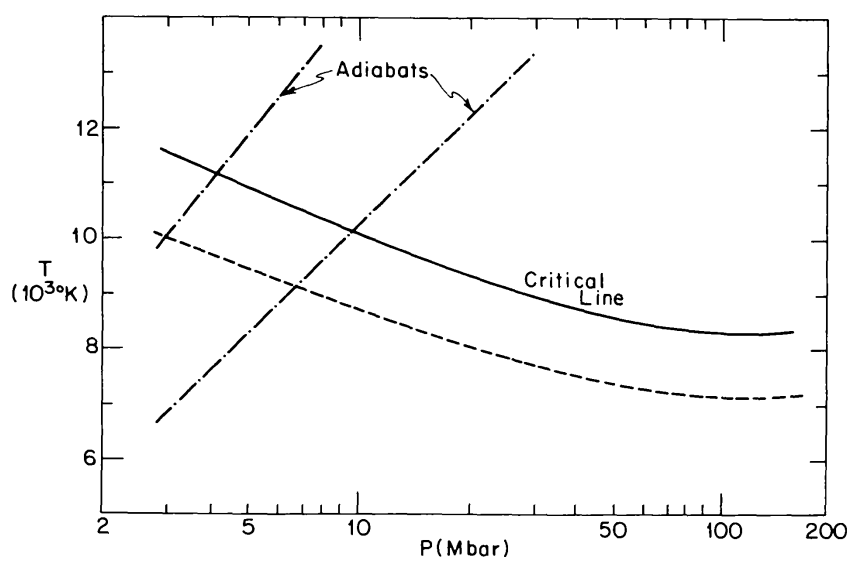

FIG. 3.-Critical line for immiscibility in a metallic $\mathbf{H}-\mathrm{He}$ mixture. Also shown (--) is the temperature below which a solar composition mixture $(10 \% \mathrm{He}$ by number) would phase separate, and two typical adiabats (-...-) appropriate to Jupiter or Saturn. 
Calculations to second-order in the electron-ion interaction (Hansen and Vieillefosse 1976; Firey and Ashcroft 1976) confirm the general features of the phase diagram, but predict somewhat lower critical temperatures. The existence of a miscibility gap can be explained merely by consideration of the Madelung energy (the electrostatic energy of the point ions immersed in a uniform electron gas), although correct allowance for the nonuniformity of the electron gas appears to increase the gap. The Madelung energy $E_{\mathrm{M}}$ can be adequately approximated by assuming ionsphere charge averaging (Salpeter 1954), according to which $E_{\mathrm{M}}$ at constant electron density is a linear function of ionic concentration. However, the comparison of alloy and separated phases must be made at constant pressure, and Stevenson $(1976 b)$ shows that under this constraint, there is a nonlinear dependence of $E_{\mathrm{M}}$ on ionic concentration such that the alloy is unfavorable relative to the separated phases. The crucial point is that at the densities and pressures of interest, the pressure is not just the Fermi contribution (independent of composition), but also has a substantial (negative) contribution from $E_{\mathrm{M}}$. At much higher pressures (for which the electron gas is relativistic) the miscibility gap may no longer exist, since constant pressure and constant electron density become equivalent (Dyson 1971; Witten 1974). In Figure 3, Madelung energy considerations dominate for $P \geqslant 10^{2}$ Mbar, whereas the rise in the critical temperature at lower pressures is explained by higherorder effects (the nonuniformity of the electron gas).

Pollock and Alder (1977) agree with the above conclusions in the high-pressure limit $\left(P \geqslant 10^{2} \mathrm{Mbar}\right)$, but conclude that at the lower pressures relevant to Jupiter, helium may be highly soluble (perhaps soluble in all proportions). However, this conclusion is based on very crude models for the low-density interactions, and it is possible to construct physically realistic models which predict that the helium solubility is least at zero pressure and increases monotonically with pressure for $0 \leqslant P \leqslant 10^{2}$ Mbar. More needs to be known about the electronic structure of helium dissolved in low-density metallic hydrogen before firm conclusions can be reached for the solubility at the lowest pressures. We shall adopt the working hypothesis that helium is least soluble in metallic hydrogen at the lowest pressure of interest (i.e., at the molecular-to-metallic hydrogen transition), and that phase separation begins for $T \leqslant 10,000 \mathrm{~K}$ at this pressure.

Solid hydrogen-helium alloys have been considered by Straus, Ashcroft, and Beck (1977). Their calculations indicate an even larger miscibility gap in the solid state than in the fluid state. This suggests that the liquidus for the alloy is lower than at least one of the melting temperatures for the pure phases, at all compositions. This effect of alloying on the melting temperature was suggested by Smoluchowski (1971) on the basis of known trends in metallic alloys. It follows that the metallic core of the giant planets is fluid (see Paper II).

\section{MOLECULAR HYDROGEN-HELIUM MIXTURES}

In contrast to the metallic state, the molecular state is not readily amenable to first-principles calculations, and we are forced to resort to semiempirical pair potentials that are compatible with experimental data, yet are also plausible modifications of first-principles calculations. Experiments have been conducted on molecular $\mathrm{H}_{2}-\mathrm{He}$ mixtures for pressures up to 7 kilobars, and a miscibility gap has been observed (Streett 1973). The calculation about to be described for megabar pressures can only be suggestive, and is not as quantitatively reliable as the metallic calculation reviewed in the previous section.

The Helmholtz free energy $F$ was calculated by Stevenson (1976a) as a function of density, temperature, the fraction $x$ (the number of molecules) of $\mathrm{He}$ in the fluid $\mathrm{H}_{2}-\mathrm{He}$ mixture. Two different calculations were carried out, one using a simple exponential 6-8 form for all the interaction potentials, with the coefficients for the $\mathrm{H}_{2}-\mathrm{H}_{2}, \mathrm{H}_{2}-\mathrm{He}$, and $\mathrm{He}-\mathrm{He}$ interactions taken from Ross (1974), Shafer and Gordon (1973), and Trubitsyn (1967), respectively. This calculation was carried out for all pressures from $1 \mathrm{kbar}$ up to $5 \mathrm{Mbar}$. The second calculation used LennardJones 6-12 potentials and was carried out only at low pressures. From $F$, the Gibbs free energy $G(P, T, x)$ was then obtained. For each pressure $P$, the requirement $\partial^{2} G / \partial x^{2}=\partial^{3} G / \partial x^{3}=0$ gives the critical temperature $T_{c}$ and the critical helium mole fraction $x_{c}$. The calculated results for $T_{c}(P)$ are given in Figure 4 and agree fairly well with Streett's experimental results, especially with regard to slope. The calculated ratio $k_{B} T_{c} / G_{e}(P)$, where $G_{e}$ is the nonideal gas part of the Gibbs free energy of the critical mixture, varies by only $50 \%$ as the pressure changes by two orders of magnitude. The slopes of the curves for $G_{e}(P)$ and $T_{c}(P)$ are probably fairly reliable, and, in view of the agreement with the experimental data at low pressures,

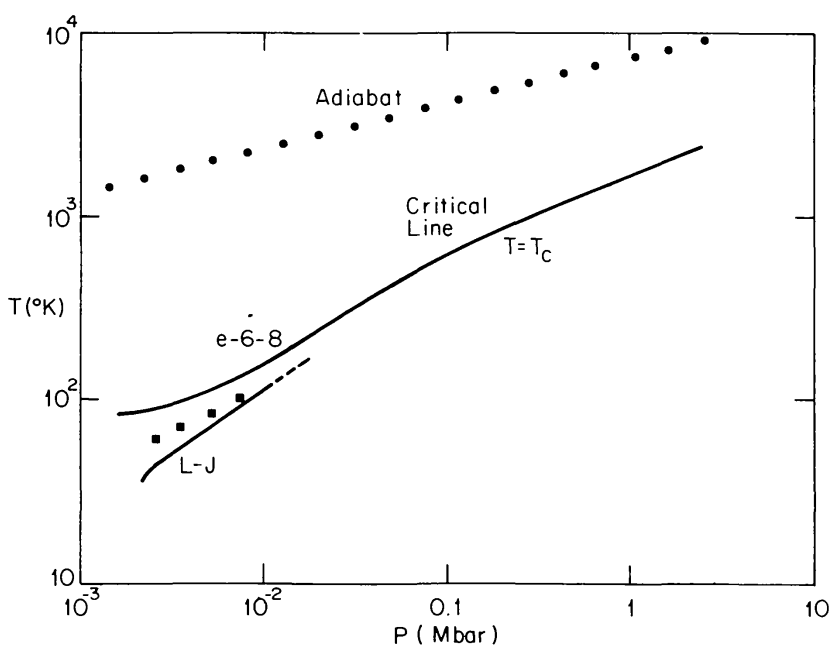

FIG. 4.-Critical line for immiscibility in a fluid $\mathrm{H}_{2}-\mathrm{He}$ mixture, for exp 6-8 and L-J potentials. Also shown are Streett's experimental critical values $(\square)$ and a typical Jovian adiabat $(\bullet \bullet \bullet)$. 
the critical curve in Figure 4 is better than an order-ofmagnitude estimate and perhaps within a factor of 2 of the correct value. The calculated value for the critical helium mole fraction was $x_{c} \approx 0.55$ at pressures appropriate to Streett's experiment, close to the experimental value of $x_{c} \approx 0.58$. The calculated value changed little with pressure, decreasing to $x_{c} \approx 0.50 \pm$ 0.05 at $P=3$ Mbar.

To summarize: If the intermolecular potentials can all be written in the simple form chosen, then Streett's experimental results have implications for the phase diagram at megabar pressures. It seems likely that at $P \approx 3 \mathrm{Mbar}, 2000 \mathrm{~K} \leqslant T_{c} \leqslant 6000 \mathrm{~K}$. This is at least a factor of 2 smaller than the critical temperature of the metallic mixture at $P \approx 3$ Mbar.

A notable feature of both Streett's experimental results and the above fluid-state calculations is that $T_{c}$ is very similar to the melting point of either pure phase. The eutectic temperature may be substantially lower, but there is nevertheless uncertainty as to whether fluid-state calculations are relevant. No solidstate calculation has been attempted for the mixture, and all subsequent considerations are confined to the fluid state. This is justified in our discussions in Paper II, since only the evolution prior to immiscibility in the molecular phase is considered in detail.

\section{THE TOTAL PHASE DIAGRAM}

The previous three sections have dealt with three aspects of the hydrogen-helium phase diagram as though they were distinct and unrelated. We now unify these into a single, coherent topology for the three-dimensional phase diagram (the dimensions being pressure $P$, temperature $T$, and composition $x$ ) according to the following model.

We consider an arbitrary hydrogen-helium mixture as a constrained ternary system of $N$ protons and helium atoms, in which $x N$ particles are helium atoms, $(1-x) y N$ are unbound protons, and $(1-x)(1-y) N$ are protons bound together as $\mathrm{H}_{2}$ molecules. The Gibbs energy of the system is approximated as

$$
G(P, T)=N\left[\sum_{i} x_{i} G_{i}^{(1)}(T)+\frac{1}{2} \sum_{i, j} x_{i} P_{i j} G_{i j}^{(2)}(P)\right],
$$

where $i$ ranges from 1 to 3 , and $x_{i}$ is the number fraction for each of the three species $(i=1$ is $\mathrm{He}$, $i=2$ is $\mathrm{H}^{+}, i=3$ is bound protons). $P_{i j}$ is the probability that a particle of species $i$ will have a particle of species $j$ as one of its nearest neighbors. The $G_{i}^{(1)}$ incorporate the ideal entropy of mixing and any chemical potential relative to an arbitrarily chosen energy zero. In other words,

$$
\begin{aligned}
G_{1}^{(1)} & =k_{B} T \ln (x / s), \\
G_{2}{ }^{(1)} & =k_{B} T \ln [(1-x) y / s]+\frac{1}{2} D, \\
G_{3}{ }^{(1)} & =\frac{1}{2} k_{B} T \ln [(1-x)(1-y) / 2 s], \\
s & \equiv x+(1-x) y+\frac{1}{2}(1-x)(1-y),
\end{aligned}
$$

where $D$ is the dissociation energy of the hydrogen molecule. Entropy effects (other than the ideal entropy of mixing) are omitted in these expressions, since thermal contributions are minor perturbations in cold systems (these entropy perturbations can be readily reintroduced for evaluating thermal derivatives along phase boundaries). The diagonal elements of $G_{i j}{ }^{(2)}$ are known since they correspond to the three pure phases (see $\S$ II). The three distinct off-diagonal elements are found by assuming numerical values for the three distinct critical temperatures $T_{c}(\mathrm{H}-\mathrm{He}), T_{c}\left(\mathrm{H}_{2}-\mathrm{He}\right)$, and $T_{c}(\mathrm{H}-\mathrm{He})$. For example, $T_{c}(\mathrm{H}-\mathrm{He})$ is the solution of $\partial^{2} G / \partial x^{2}=\partial^{3} G / \partial x^{3}=0$ for $y \equiv 1$. A random mixture was assumed, so that $P_{i j}=x_{j} / s$. This simple choice automatically implies the following simple compositions for the critical mixtures: $x_{c}=1 / 2$ for $\mathrm{H}-\mathrm{He}, x_{c}=1 / 3$ for $\mathrm{H}_{2}-\mathrm{He}$ (half $\mathrm{H}_{2}$, half $\mathrm{He}$ ), and $y=1 / 3$ for $\mathrm{H}-\mathrm{H}_{2}$ (half $\mathrm{H}_{2}$, half $\mathrm{H}$ )-all crude but adequate approximations. The total Gibbs energy for a given $x, P$, and $T$ is then minimized with respect to $y$ to yield the equilibrium state of the hydrogen. At sufficiently low temperatures there are two minimaone corresponding to "metallic" hydrogen, the other corresponding to "molecular" hydrogen. Except in special cases, one minimum will be lower than the other and correspond to the equilibrium state. The higher minimum corresponds to the metastable state. If the temperature is too high, or the helium content is too great, then the first-order character of the molecular-metallic transition is "washed out," and there is only one minimum.

For each $(P, T)$ the existence of one or more common tangents to the equilibrium Gibbs energy as a function of $x$ determines the coexisting phases and the thermodynamically inaccessible regions. In this way, the phase diagram was mapped out for all $P, T$, $x$ of interest.

We shall describe in detail the results for the choice

$$
\begin{aligned}
T_{c}(\mathrm{H}-\mathrm{He}) & =12,000 \mathrm{~K}, \\
T_{c}\left(\mathrm{H}_{2}-\mathrm{He}\right) & =6,000 \mathrm{~K}, \\
T_{c}\left(\mathrm{H}_{-} \mathrm{H}_{2}\right) & =18,000 \mathrm{~K},
\end{aligned}
$$

which, according to the discussion of the previous sections, is a possible selection. (For simplicity, the pressure dependence of each $T_{c}$ is ignored.) Figure 5 illustrates the results. Consider, first, diagram $(a)$, for which $T=13,000 \mathrm{~K}$. At each pressure in the range 3-4.6 megabars there coexist a helium-poor metallic phase and a helium-rich molecular phase whenever the total helium content lies within the shaded region. Below the dashed line, the metallic phase is more dense than the molecular phase, whereas the reverse is true above the dashed line. This "density inversion" is a consequence of the competition between the density increase accompanying the addition of helium, and the density decrease accompanying the metallicmolecular transition. At sufficiently large helium concentration $x$, the first-order character of the metallic-molecular transition is lost and there are no excluded regions. 


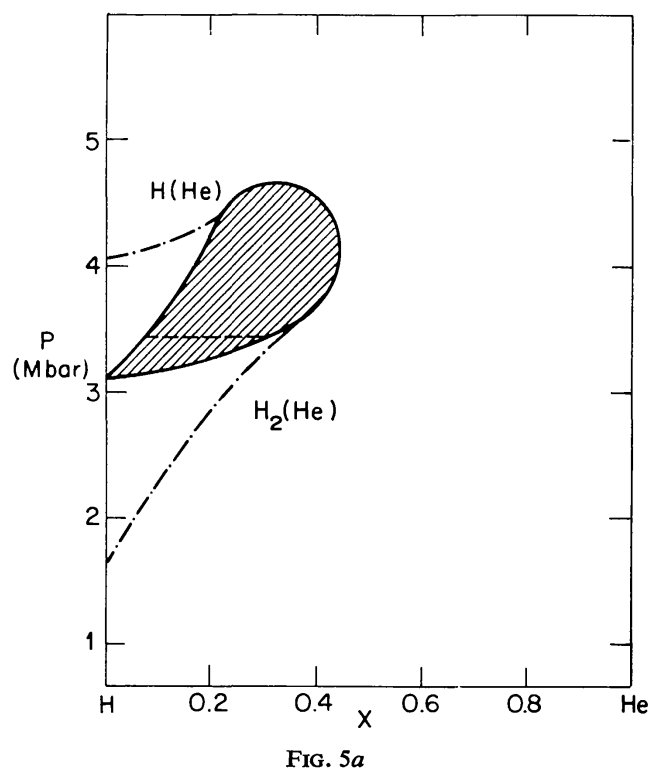

FIG. 5.-Phase diagrams for three different temperatures: (a) $T=13,000 \mathrm{~K},(b) T=7500 \mathrm{~K}$, (c) $T=4000 \mathrm{~K}$. In each case, the phase-excluded region is shaded. Above the dashed line (---), the phase on the right-hand side of the phaseexcluded region has greater mass density than the coexisting phase on the left-hand side. Below the lower dot-dashed curve (..-) the metallic phase ceases to be metastable. Above the upper dot-dashed curve the molecular phase ceases to be metastable. Note the presence of a triple point $A$ in diagram (c).

Consider diagram $(b)$ of Figure 5. Since $T=$ $7500 \mathrm{~K}<T_{c}(\mathrm{H}-\mathrm{He})$, there is now a miscibility gap which extends to high pressures. This evolves smoothly from the "loop" of diagram $(a)$. Notice that there is no clear distinction between the molecular-metallic transition and the phase separation in the metallic fluid. Proceeding smoothly along the lower phase

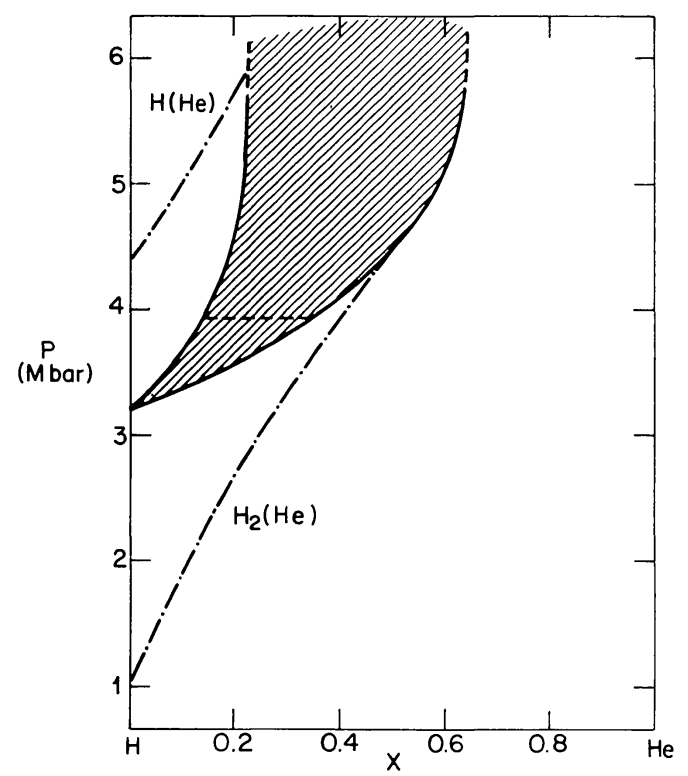

Fig. $5 b$

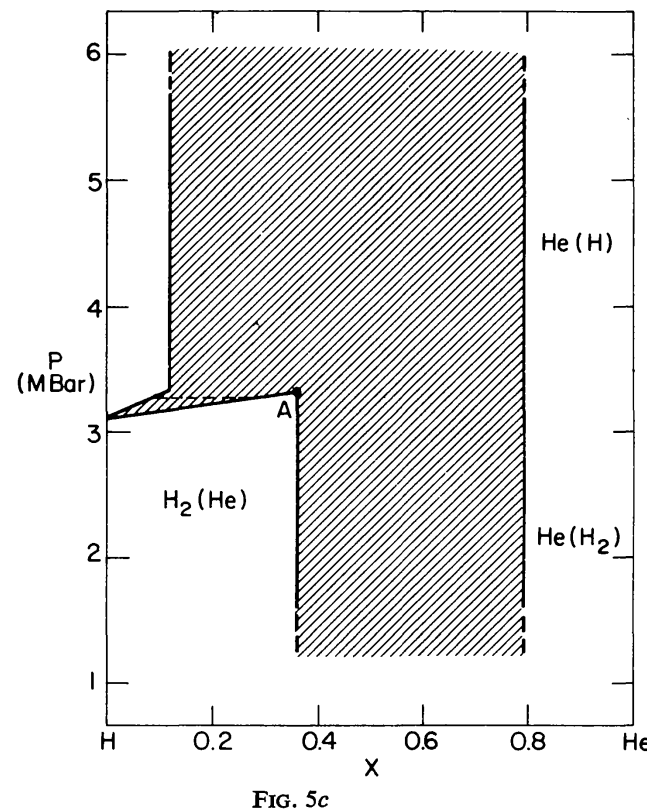

boundary from small $x$ to large $x$, the fluid progresses smoothly from predominantly molecular to predominantly metallic.

In diagram $(c), T=4000 \mathrm{~K}$ and there is now a miscibility gap in the molecular fluid. This miscibility gap forms smoothly from diagram $(b)$, as $T$ is lowered, in the following way: At some critical temperature, $T_{c}{ }^{*}$, an inflection becomes formed in the lower phase boundary of diagram $(b)$. In this model, $T_{c}{ }^{*}$ is comparable to $T_{c}\left(\mathrm{H}_{2}-\mathrm{He}\right)$. For $T<T_{c}{ }^{*}$ a minimum in $P$ (as a function of $x$ along the phase boundary) is formed, and the miscibility gap rapidly grows as $T$ is further reduced. Immediately below $T_{c}{ }^{*}$ a triple point [marked $A$ in diagram $(c)$ ] is formed. Thus there is a line of triple points ending at a critical point $T=T_{c}$ * (at $P \approx 3.5 \mathrm{Mbar}$ ). The concentration at the triple point is a sensitive function of temperature, and becomes smaller as the temperature is reduced and the excluded region expands to fill most of $(P, x)$-space. At low temperatures, the "density inversion" effect eventually vanishes and the immiscibility effects dominate.

For general values of the parameters in equation (7) one can define a "configuration space" in which each point is itself a phase diagram. This is shown in Figure 6 for the choice $T_{c}(\mathrm{H}-\mathrm{He})=2 T_{c}\left(\mathrm{H}_{2}-\mathrm{He}\right)$. For given values of $T_{c}(\mathrm{H}-\mathrm{He}), T_{c}\left(\mathrm{H}-\mathrm{H}_{2}\right)$, and $T$ one can find from this "configuration" diagram what the topology of the physical phase diagram is.

In the following paper (Paper II) these model phase diagrams will be used in considering specific compositional and thermal histories of an evolving hydrogenhelium planet such as Jupiter.

\section{MINOR CONSTITUENTS}

It is clear both from atmospheric observations and interior models that the hydrogen-helium planets 


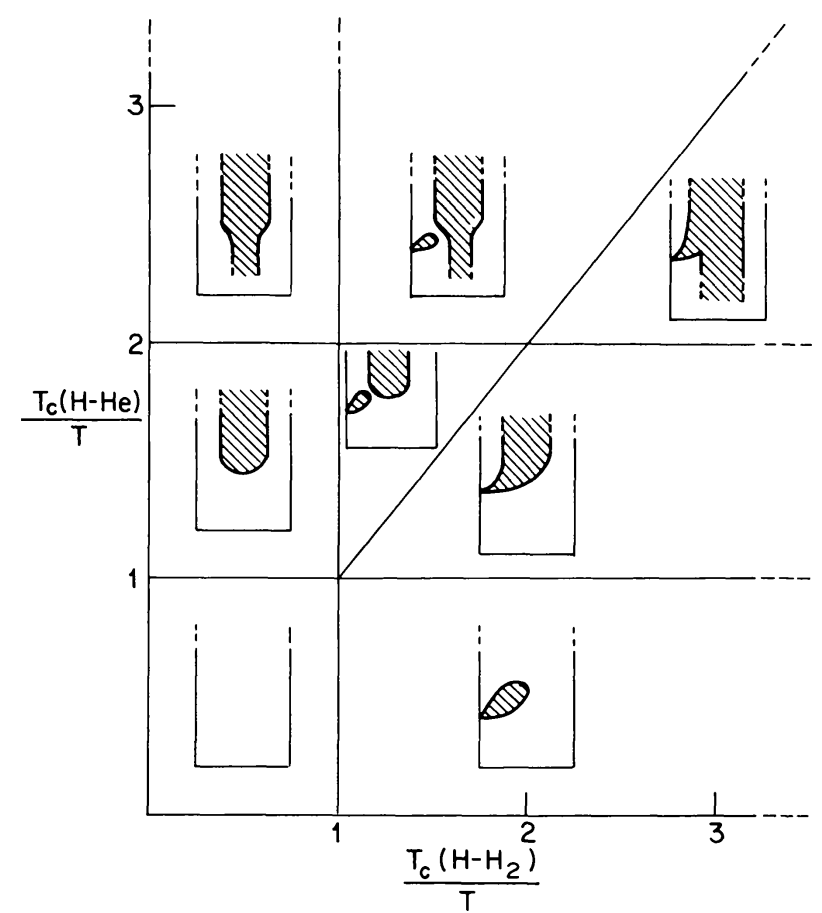

FIG. 6.-Various possible phase diagrams, assuming $T_{c}(\mathrm{H}-\mathrm{He})=2 T_{c}\left(\mathrm{H}_{2}-\mathrm{He}\right)$. Each small diagram within the figure is a schematic representation of a $(P, x)$-diagram similar to that in Fig. 5 .

contain minor constituents at least to the extent of solar abundance. The distribution of these minor constituents is important both for model construction and for relating the observed atmospheric abundance to the total abundance. There is the possibility that an appropriately chosen minor constituent or group of constituents could be very precise "tracers" of internal dynamic processes by virtue of their almost complete partitioning into one of the hydrogen-helium phases. No especially appropriate tracer is indicated by the analysis of this section, which deals primarily with general trends. The special case of deuterium is discussed separately. This section deals only with thermodynamic considerations. The actual distribution of constituents within an evolving planet also depends on fluid-dynamic and diffusive processes (Paper II).

\section{a) Deuterium}

Both $\mathrm{CH}_{3} \mathrm{D}$ (Beer et al. 1972) and HD (Trauger et al. 1973) have been observed in the Jovian atmosphere, and the inferred deuterium abundance has been frequently quoted as indicative of the primordial solar (or even cosmic) abundance. The partitioning of deuterium therefore has an importance out of proportion to its abundance. Unlike other minor constituents, the chemical potential of deuterium is readily calculable (as a simple extension of the analysis of ordinary hydrogen).

Consider, first, the partitioning of a small amount of deuterium between pure, coexisting molecular and metallic phases of ordinary hydrogen. Hubbard
(1974) concluded that the mass fraction of deuterium in the metallic phase exceeds that in the molecular phase by roughly $15 \%$. His calculation is for the "classical" (i.e., high-temperature) limit but neglects the vibrational degrees of freedom for the $\mathrm{H}_{2}$ and $\mathrm{HD}$ molecules, and also neglects dissociation. If, instead, one assumes that the vibrational degrees of freedom are fully excited and harmonic, then the chemical constant of HD is increased by $\frac{1}{2} \ln \left(\frac{4}{3}\right)$ relative to $\mathrm{H}_{2}$, and the mass fraction of deuterium in each phase is exactly the same. (This is a general result for the classical limit and not a special property of hydrogen.) Excitation of the vibrational modes probably is achieved at $10^{4} \mathrm{~K}$, the temperature of interest, since the low-density vibrational temperature for $\mathrm{H}_{2}$ is $6000 \mathrm{~K}$, and this does not appear to increase at high density (Silver and Stevens 1973). As the temperature is reduced, another effect not considered by Hubbard becomes important: quantum corrections to the translational energy of the protons and deuterons in the metallic state. This can be calculated from the Wigner theory as in Stevenson (1975). This positive contribution to the chemical potential is larger for protons than for deuterons and therefore favors partitioning of deuterons into the metallic phase. (The competing quantum effect in the molecular phase is negligible.) The incomplete excitation of the vibrational modes of $\mathrm{H}_{2}$ and $\mathrm{HD}$ also favors partitioning into the metallic phase. Numerical calculations indicate that the mass ratio of deuterium (metallic) to deuterium (molecular) is essentially unity for $T \geqslant$ $8000 \mathrm{~K}$, about 1.05 at $T \approx 5000 \mathrm{~K}$, and 1.25 at $T \approx 2500 \mathrm{~K}$.

Consider now the partitioning of deuterium between hydrogen-rich and helium-rich metallic phases. In the relevant high-temperature limit, the only free energy contribution tending to produce a partitioning of deuterons different from the partitioning of protons is the quantum translational energy. According to the Wigner theory, the shift in equilibrium is such as to favor less variation of the ionic thermal de Broglie wavenumber. The deuterium-to-hydrogen ratio is thus greater in the helium-rich phase. Numerical calculation, based on the evaluation of $F_{Q}$ in Stevenson (1975), indicates that this ratio is $10 \%$ larger in the helium-rich phase than in the hydrogen-rich phase at $T=5000 \mathrm{~K}$, with the difference vanishing at $T \approx 10,000 \mathrm{~K}$.

The deuterium-to-hydrogen ratios in coexisting hydrogen-rich and helium-rich molecular phases should coincide at the temperatures of interest, provided the rotational and vibrational degrees of freedom of the $\mathrm{H}_{2}$ and $\mathrm{HD}$ molecules are not strongly influenced by the fraction of helium in the local environment. In the absence of a detailed model for these modes, no quantitative calculation can be made. Substantially unequal partitioning seems unlikely, however.

In conclusion, the partitioning of deuterium between the various hydrogen-helium phases appears to preserve the deuterium-to-hydrogen mass ratio, at least for $T \geqslant 5000 \mathrm{~K}$. The deuterium content in the 
uppermost convective layers of hydrogen-helium planets should therefore be representative of the bulk composition, provided the reservoir of material from which the planet formed had a uniform distribution of deuterium.

\section{b) Other Minor Constituents}

First, consider the possibility of a phase transition caused by a minor constituent (e.g., insolubility of a minor constituent). This could occur independently of the existence of phase boundaries in the hydrogenhelium, but it is improbable for the low concentrations and high temperatures of interest. If the number fraction of a minor constituent is $z$, then an energy of about $-k_{B} T \ln z$, which favors the dissolved state, must be compensated by an effect which favors the separated phase. For example, water at $T \leqslant 300 \mathrm{~K}$, pressures of order of a few bars, and abundance $z \approx 10^{-3}$ can preferentially form droplets since $-k_{B} T \ln z \leqslant 0.2 \mathrm{eV}$ can be overcome by the binding energy of the liquid water. In the deep interior of the planet, however, $-k_{B} T \ln z \approx 6 \mathrm{eV}$, and there is apparently no correspondingly large binding effect. Water is probably insoluble in molecular hydrogen at low enough temperatures or high enough concentrations, but this is probably not relevant to the deep interiors of present giant planets. We shall therefore restrict ourselves to a discussion of partitioning between phases of the hydrogen-helium system.

The degree of partitioning is determined by equating the chemical potentials for the impurity in the two coexisting phases. At high pressures, the chemical potential can be meaningfully separated into four parts: (i) the "nonchemical" electronic contribution (i.e., a part which does not explicitly invoke the symmetry properties or discreet band structure of the electronic spectrum); (ii) residual chemical effects [i.e., electronic effects not included in (i)]; (iii) configurational (including entropy) effects, resulting from the different size of solute and solvent atoms; and (iv) the ideal free energy of mixing.

Consider first the "nonchemical" electronic contribution. In the high-pressure limit, where the electrons can be considered to be a uniform Fermi gas, Stevenson $(1976 b)$ showed that the miscibility gap in a binary alloy increases as the difference between the nuclear charges of the constituents increases. A direct corollary of this result is that ions will partition so as to minimize nuclear charge differences. Thus all elements with $Z \geq 3$ will preferentially partition into the helium-rich phase of a hydrogen-helium mixture. A more general result, applicable to lower pressures, can be obtained by an extension of the Thomas-FermiDirac (TFD) method. The usual TFD procedure for an alloy is to assume volume additivity, whereby the locally evaluated "pressure" at the Wigner-Seitz cell boundary is assumed to be the same for every cell. If electron correlation is ignored, or evaluated in a local approximation, then this also implies continuity of the electron density across cell boundaries (Salpeter and Zapolsky 1967). Clearly, this procedure predicts that the chemical potential of a constituent is independent of its environment (at a given pressure) so that no nonuniform partitioning could occur. The failure of the TFD method is not so much in the prescription for determining the charge density (which is very accurate at sufficiently high pressure) but in the unphysical procedures for evaluating pressure and assigning boundary conditions. We propose that a better, albeit more complicated, procedure is to enforce continuity of the electron density at the cell boundaries, and calculate pressure according to the rigorous (i.e., nonlocal) thermodynamic derivative of the total energy with respect to volume. Let $\rho \equiv$ $\rho(P)$ be the actual electron density at the WignerSeitz cell boundary (approximated by a sphere) at pressure $P$. Let $V_{i}(\rho)$ be the specific (cell) volume of species $i$, and $E_{i}(V)$ be the energy per cell (evaluated as though the substance were purely species $i$ ). In accord with the Wigner-Seitz philosophy, the total energy per atom is assumed to be

$$
E=\sum_{i} x_{i} E_{i}\left\{V_{i}[\rho(P)]\right\},
$$

where $x_{i}$ is the number fraction of species $i$. [The energy is not a linear function of the $x_{i}$, since $\rho(P)$ is also a self-consistently determined function of the alloy composition.] It then follows that in the limit of vanishing concentration for species $i$, the chemical potential $\mu_{i}$ is

$$
\begin{aligned}
\mu_{i} & =\mu_{i}^{0}+\Delta \mu_{i}, \\
\mu_{i}^{0} & =E_{i}\left\{V_{i}\left[\rho_{i}(P)\right]\right\}+P V_{i}\left[\rho_{i}(P)\right], \\
\Delta \mu_{i} & =-\frac{1}{2}\left(\frac{\partial P}{\partial V}\right)_{i}\left[\left.\left(\rho_{0}-\rho_{i}\right) \frac{\partial V_{i}}{\partial \rho}\right|_{\rho_{i}}\right]^{2},
\end{aligned}
$$

to lowest nonvanishing order in $\left(\rho_{0}-\rho_{i}\right)$, where $\rho_{i}(P)$ is the cell boundary electron density for a pure substance composed of species $i$, and $\rho_{0}(P)$ is the cellboundary electron density for the solvent phase (the relevant hydrogen-helium phase in this case). The TFD procedure (without correlation or with locally evaluated correlation) predicts $\rho_{0} \equiv \rho_{i}$ and $\Delta \mu_{i} \equiv 0$. The above procedure does not require that the $E_{i}(V)$ be evaluated according to TFD and, in general, $\rho_{0} \neq \rho_{i}$. The $\Delta \mu_{i}$ is always positive, and can be regarded as a microscopic "surface energy." The model predicts that a solute preferentially enters the phase in which the cell boundary electron density is most compatible. For example, $\rho(\mathrm{He})$ is more similar to $\rho\left(\mathrm{H}_{2}\right)$ than $\rho$ (metallic $\left.\mathrm{H}\right)$, and helium therefore prefers the molecular phase, in accord with our discussion in $\S \mathrm{V}$.

Unfortunately, the pressure of interest is not high enough for simple generalities based only on nuclear charge. For example, $\mathrm{Na}$ and $\mathrm{Al}$, elements with similar nuclear charges, behave quite differently. Pseudopotential theory (with polarizable core states) suggests that the essentially monovalent $\mathrm{Na}$ has $\rho \approx 0.041 a_{0}{ }^{-3}$ at $P=3 \mathrm{Mbar}$ ( $a_{0}$ is the first Bohr radius), whereas the trivalent $\mathrm{Al}$ has $\rho \approx 0.058 a_{0}{ }^{-3}$. (For a discussion of 
pseudopotential theory, see Ashcroft and Langreth 1967.) The corresponding cell boundary densities for hydrogen are $0.06 a_{0}^{-3}$ (metallic) and $0.035-0.04 a_{0}{ }^{-3}$ (molecular). The metallic value is estimated from Wigner-Seitz calculations (Neece, Rogers, and Hoover 1971) and the molecular value from band structure calculations (Friedli and Ashcroft 1976). If metallic hydrogen is the solvent, then (from eq. [9]), $\Delta \mu_{\mathrm{Na}} \approx$ $2 \mathrm{eV}$ and $\Delta \mu_{\mathrm{Al}} \approx 0$; whereas if molecular hydrogen is the solvent, then $\Delta \mu_{\mathrm{Na}} \approx 0$ and $\Delta \mu_{\mathrm{Al}} \approx 1.5 \mathrm{eV}$. If other factors were negligible then Al would prefer metallic hydrogen and helium-poor phases, whereas $\mathrm{Na}$ would prefer molecular hydrogen and heliumrich phases. Further generalization is difficult, and the partitioning of $\mathrm{Fe}$ and $\mathrm{Mg}$ (for example) is not readily predicted. One would expect, however, that atoms or molecules with closed shell configurations at low densities would, in most instances, still have low cell boundary electron densities even at megabar pressures, and prefer molecular or helium-rich phases. This might include the abundant "closed shell" species $\mathrm{H}_{2} \mathrm{O}, \mathrm{CH}_{4}$, and $\mathrm{NH}_{3}$ (but see the discussion on $\mathrm{H}_{2} \mathrm{O}$ at the end of this section).

Consider, now, the "chemical" effects that are not implicit in the previous analysis. These are difficult to estimate, but appear to be small. For example, it might be supposed that a metal would not dissolve in dense molecular hydrogen because the available conduction states in the hydrogen are separated from the valence band by an energy gap. However, the band gap is $\leqslant 1 \mathrm{eV}$ at the transition pressure (Friedli and Ashcroft 1976), so this effect may be less than that predicted by equation (9). Similarly, the categorization of polar and nonpolar molecules is meaningless at megabar pressures, and the distinctions among covalent, ionic, and metallic bonding become inapplicable.

The configurational contribution to the chemical potential can be estimated for the fluid phase by the hard sphere model (Lebowitz and Rowlinson 1964), with the effective (pressure- and temperaturedependent) hard sphere diameters determined by minimization of the total free energy. Numerical calculations indicate that this contribution is several $k_{B} T$ at $T \approx 10^{4} \mathrm{~K}$, but that the difference between solute potentials for the various solvent phases is less than $k_{B} T \approx 1 \mathrm{eV}$ and therefore usually small compared with electronic differences.

The ideal free energy of mixing is $k_{B} T \ln z$, where $z$ is the number fraction of the solute. Typically, the electronic chemical potential differences between two coexisting phases are a few $\mathrm{eV}$, so that for $k_{B} T \approx 1 \mathrm{eV}$ the value of $z$ could change by as much as an order of magnitude as one crosses a phase boundary.

We conclude with a brief discussion of the partitioning of $\mathrm{H}_{2} \mathrm{O}$, probably the most abundant minor constituent in Jupiter and Saturn (although possibly underabundant in the Jovian atmosphere, according to Larson et al. 1975). According to the preceding analysis, we would expect $\mathrm{H}_{2} \mathrm{O}$ to prefer molecular and helium-rich phases. However, this assumes that the configuration-and the electronic structure-of
$\mathrm{H}_{2} \mathrm{O}$ is similar for each phase. Pure water is completely dissociated into $\mathrm{H}_{3} \mathrm{O}^{+}$and $\mathrm{OH}^{-}$at about 200 kilobars (Hamann and Linton 1966) and is metalized at several megabars (Ramsey 1963; Vereschchagin, Yakovlev, and Timofeev 1975b), at which pressure nothing is known about the configuration. The dissociation does not significantly modify the previous analysis, since $\mathrm{H}_{3} \mathrm{O}^{+}$and $\mathrm{OH}^{-}$are both isoelectronic with a closed shell atom (neon). However, one should consider the possibility that $\mathrm{H}_{2} \mathrm{O}$ enters metallic hydrogen as $2 \mathrm{H}^{+}+\mathrm{O}^{n+}+(n+2) e^{-}$, where $n \geq 0$. Approximate numerical calculations suggest that this is highly improbable, even for $n=1$, despite the similarity of the first ionization energy of oxygen $(\sim 13.6 \mathrm{eV})$ and the binding energy per electron of the metallic state. The problem is that the energy reduction gained by "metalizing" the oxygen atom is small, and does not compensate the rather large binding energy of the $\mathrm{OH}^{-}$ ion. The chemical potential of $\mathrm{H}_{2} \mathrm{O}$ in molecular hydrogen is $\sim 20 \mathrm{eV}$ (relative to the isolated zeropressure $\mathrm{H}_{2} \mathrm{O}$ molecule), whereas the chemical potential for the hypothetical metalized state (with the oxygen in the $\mathrm{O}^{+}$form) has a chemical potential $\sim 28 \mathrm{eV}$ at least.

\section{TRANSPORT PROPERTIES OF THE METALLIC PHASE}

We consider essentially all the "first-order" atomic transport coefficients in the following order: electrical conductivity, thermal conductivity, viscosity, selfdiffusion, inter-diffusion, and radiative opacity. There is also a brief discussion of "second-order" (or offdiagonal) transport coefficients such as the Soret coefficient.

\section{a) Electrical Conductivity}

This has been evaluated by Stevenson and Ashcroft (1974) using the well-known Ziman theory, and the hard sphere static structure factors. In that paper, the temperature scale was only estimated, but subsequent thermodynamic calculations (Stevenson 1975) established the correspondence between hard sphere diameter and temperature for each density. An estimate can also be made for the dynamic corrections, using the theory of Baym (1964) and the moleculardynamics results of Hansen, McDonald, and Pollock (1975) for the one-component plasma. The improved temperature scale and the dynamic corrections each modify the results of Stevenson and Ashcroft (1974) by as much as a factor of 2-but in opposite directions. The final result is the following approximate formula for the conductivity $\sigma$ :

$$
\sigma \approx \frac{5 \times 10^{20} \rho^{4 / 3}}{T(1+3 x)} \mathrm{esu}
$$

where $\rho$ is the mass density in $\mathrm{g} \mathrm{cm}^{-3}$, and $x$ is the helium number fraction. This formula should be correct to within a factor of 2 for $1 \leqslant \rho \leqslant 10^{2} \mathrm{~g} \mathrm{~cm}^{-3}$ and $10^{3} \leqslant T \leqslant 10^{6} \mathrm{~K}$, but should only be used for $x \leqslant 0.2$. In the conditions prevailing in the Jovian 
core at present, $\sigma \approx 10^{17}$ esu, comparable to that of room-temperature alkali metals. The value of $\sigma$ given by equation (10) is about a factor of 2 larger than the estimates for solid metallic hydrogen by Abrikosov (1964) and Hubbard and Lampe (1969).

\section{b) Thermal Conductivity}

In the metallic phase, thermal conductivity is dominated by electronic transport. If the electrons are degenerate, and if the Born approximation is valid (see Stevenson and Ashcroft 1974 for a discussion of this point), then the thermal conductivity is related to the electrical conductivity by the WiedemannFranz relation. The thermometric conductivity $\kappa$ is then given by

$$
\rho C_{p^{\kappa}} \approx \frac{1.5 \times 10^{8} \rho^{4 / 3}}{(1+3 x)} \text { ergs } \mathrm{cm}^{-1} \mathrm{~s}^{-1} \mathrm{~K}^{-1},
$$

or, if we assume $C_{p} \approx 3 N k_{B}$, where $N$ is the number of ions per gram,

$$
\kappa \approx 0.3 \rho^{1 / 3} \mathrm{~cm}^{2} \mathrm{~s}^{-1} .
$$

Notice that the temperature $T$ does not appear in equations (11) and (12). The accuracy and validity of these equations is the same as for the electrical conductivity.

\section{c) Viscosity}

Unlike the electronic transport properties above, viscosity and atomic diffusion depend explicitly on the dynamic properties of the fluid. There is no generally accepted and successful theory for the dynamics of a dense fluid. However, models which work for the conventional alkali metals, such as the LonguetHiggins and Pople (1956) model, as adapted by Ascarelli and Paskin (1968) and modified by Vadovic and Colver (1971), probably are also satisfactory for metallic hydrogen. The following approximate formula is then deduced:

$$
\nu \approx 4 \times 10^{-3} T_{4}^{-1 / 2} \mathrm{~cm}^{2} \mathrm{~s}^{-1},
$$

for any hydrogen-helium mixture, where $T_{4}$ is the temperature in units of $10^{4} \mathrm{~K}$. The apparent lack of density dependence in this result is only approximate. At the temperatures and densities of interest, this result should be correct to at least a factor of 5 (and probably a factor of 2 ).

This calculation is based on a hard sphere approach. The opposite extreme is the one-component plasma, which can be regarded as the unscreened metallic state. Two calculations for this system (Hansen, McDonald, and Pollock 1975; Vieillefosse and Hansen 1975) agree that

$$
\nu \approx 0.1 \omega_{p} \bar{r}^{2}
$$

to within a factor of 2 , where $\omega_{p}$ is the ion plasma frequency and $\bar{r}$ is the radius of that sphere which contains one ion on the average. This formula yields a value that is typically a factor of 2 smaller than equation (13), at least for $T_{4} \approx 1$, and it also predicts a very weak density dependence $\left(\nu \propto \rho^{-1 / 6}\right)$.

From equations (12) and (13), we can now estimate the Prandtl number Pr:

$$
P r \equiv \frac{\nu}{\kappa} \approx 10^{-2} T_{4}^{-1 / 2} \rho^{-1 / 3},
$$

provided the helium content satisfies $x \leqslant 0.2$. (Heliumrich fluids may have a substantially lower $\kappa$.) Thus, for $T_{4} \approx 1$ and $\rho \approx 1 \mathrm{~g} \mathrm{~cm}^{-3}, P r \approx 10^{-2}$, which is typical of liquid alkali metals.

\section{d) Self-Diffusion}

This transport property may not be of great interest itself, but it provides a means of estimating the more interesting interdiffusion (diffusion of helium in hydrogen). We use the same theory as for the viscosity (Vadovic and Colver 1971), which predicts that the product of self-diffusion $D$ and viscosity $\nu$ is given by

$$
D \nu \approx 0.17 \sigma^{2}\left(\frac{k_{B} T}{M}\right),
$$

where $\sigma$ is the effective hard sphere diameter, and $M$ the ion mass. This result is experimentally verified when $\sigma$ is chosen by thermodynamic considerations alone. Thus,

$$
D \approx 3 \times 10^{-3} \rho^{-2 / 3} T_{4}^{3 / 2} \quad \mathrm{~cm}^{2} \mathrm{~s}^{-1},
$$

for both pure hydrogen and pure helium.

The one-component plasma studies (Hansen, McDonald, and Pollock 1975; Vieillefosse and Hansen 1975) predict $D \propto \rho^{-2 / 3} T^{4 / 3}$ and a magnitude that is typically a factor of 3 smaller than that given by equation (17). This agreement is satisfactory, and suggests that this transport property is not strongly dependent on the details of the ion-ion interaction.

\section{e) Interdiffusion}

There is no similarly successful model for interdiffusion, so we shall resort to empirical evidence. Experiments on liquid metal mixtures (Ejima and Yamamura 1973) indicate that the interdiffusion of one atomic species in another differs from the selfdiffusion of the most abundant species to the extent that the species differ in "size." Thermodynamic calculations (Stevenson 1975) indicate that the helium pseudoatom ( $\alpha$-particle plus screening cloud of electrons) is $30 \%$ larger than the hydrogen pseudoatom. The experiments then indicate that a small amount of helium in hydrogen should diffuse about half as rapidly as the self-diffusion of hydrogen. Thus

$$
D_{\text {H-Hе }} \approx 1.5 \times 10^{-3} \rho^{-2 / 3} T_{4}^{3 / 2} \mathrm{~cm}^{2} \mathrm{~s}^{-1},
$$

and independent of composition to a first approximation. 
To see whether diffusion is anomalous near a phase transition, we first express the interdiffusion coefficient $D$ in a more fundamental form (Landau and Lifshitz 1959):

$$
D=\frac{\alpha}{\rho}\left(\frac{\partial \mu}{\partial x}\right)_{T, P},
$$

where $\mu$ is the helium chemical potential, $x$ is the helium concentration, and $\alpha$ is a "canonical" kinetic coefficient, as explained by Landau and Lifshitz. The requirement that entropy increase with time implies that $\alpha>0$. Consider, now, the specific Gibbs energy in Figure 7a. (This is a schematic representation of Fig. 2 in Stevenson 1975.) Between $A$ and $D$, a fluid mixture is energetically unfavorable relative to separated helium-rich and hydrogen-rich phases. Between $A$ and $B$ and between $C$ and $D$ the fluid mixtures are metastable (i.e., $\partial^{2} \Delta G / \partial x^{2}=\partial \mu / \partial x>0$ ). In these regions, phase separation must proceed by nucleation and can be strongly inhibited by the surface energy between the phases. Between $B$ and $C$, the fluid mixture is unstable to spinodal decomposition (the onset
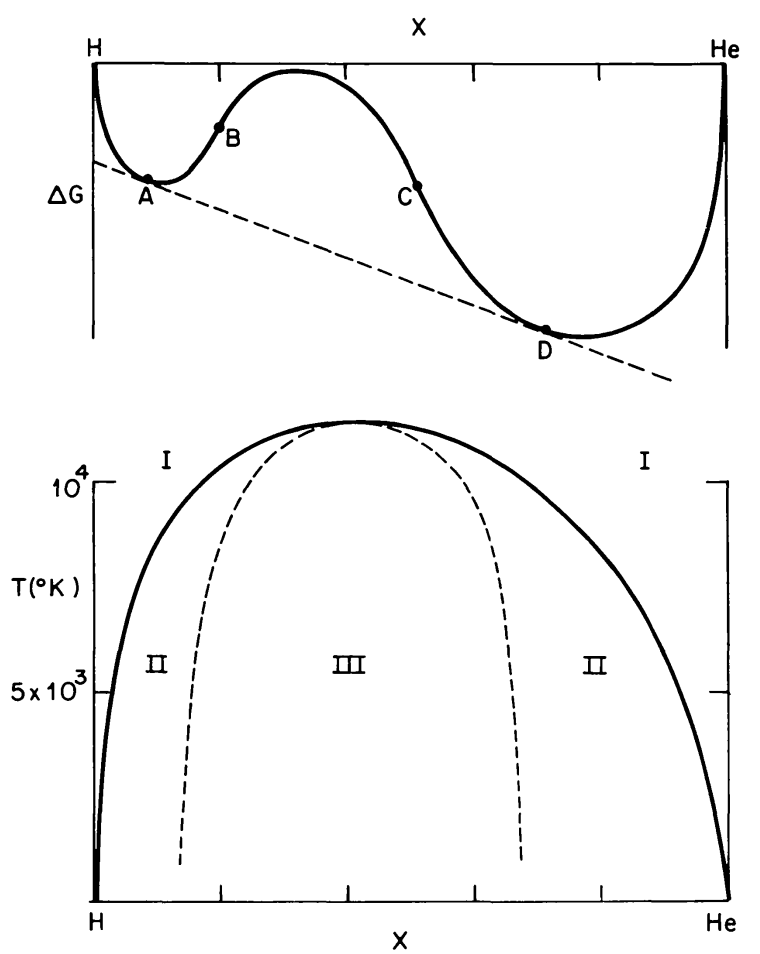

FIG. 7. (a) (top)-Gibbs energy of mixing for a $\mathrm{H}-\mathrm{He}$ mixture at a given pressure and temperature, as a function of helium concentration $x$. The dashed line is a common tangent to the Gibbs energy curve. Regions $A B$ and $C D$ correspond to metastable fluid mixtures, and the diffusion constant is not anomalous, except near $B$ and $C$. The region between $B$ and $C$ corresponds to unstable mixtures. (b) (bottom) The phase diagram of $\mathrm{H}-\mathrm{He}$ mixtures for a given pressure. In region $\mathrm{I}$ the uniform mixture is thermodynamically favored. In region II the uniform mixtures are metastable and diffusion is not anomalous. In region III the uniform mixture is unstable and undergoes spinodal decomposition. The dashed line separates regions of normal and "anomalous" diffusion. of long-wavelength concentration fluctuations), the rate of which is essentially limited only by diffusion rather than by surface energy. In this region, $\partial \mu / \partial x<0$, and the diffusion coefficient can be regarded as negative in the sense that compositional inhomogeneities tend to grow rather than decay with time. At the points $B$ and $C$, the diffusion constant is zero. In Figure $7 b$ the phase diagram for a given pressure is shown and the various regions indicated. Spinodal decomposition has recently been clearly simulated for the first time in computer experiments (Abraham et al. 1976) and has been the subject of several theoretical investigations (Abraham 1975a, b).

The important point for our considerations is that, provided one is not within or near region III in Figure $7 b$, the diffusion coefficient is not anomalous. We will return to this point in Paper II, where the dynamics of the phase separation are discussed for a real system.

\section{f) Radiative Opacity}

At the temperatures of interest $\left(T \approx 10^{4} \mathrm{~K}\right)$, thermal photons have energies of order $1 \mathrm{eV}$. At the densities of interest $\left(\rho \approx 1 \mathrm{~g} \mathrm{~cm}^{-3}\right)$, the electron plasmon energy is of order $30 \mathrm{eV}$. Photons cannot propagate below the plasmon energy and still undergo substantial absorption above the plasmon energy. It follows that the radiative opacity exceeds the electron conduction "opacity" by many orders of magnitude in the metallic phase. It can therefore be ignored.

\section{g) Second-Order Transport Coefficients}

Among the many "second-order" transport coefficients, there are those which characterize the effect of simultaneous concentration, thermal, and pressure gradients in a nonconvecting fluid. First, there is the barodiffusion caused by the pressure gradient. In the applications to be discussed in Paper II, the composition varies over a smaller length scale than the pressure scale height, so the effect of barodiffusion is small. (Of course, barodiffusion does nevertheless ensure that the zero temperature final state of a selfgravitating body is inhomogeneous.) Second, there is the effect of solute flux on the thermal gradient (the DuFour effect). The Onsager reciprocal relations ensure that this effect is always negligibly small for a dense fluid (Caldwell 1973). Third, there is the effect of the temperature gradient on the solute flux $\boldsymbol{F}_{\boldsymbol{x}}$ (Landau and Lifshitz 1959),

$$
\boldsymbol{F}_{x}=-\rho D\left(\nabla x+\frac{k_{T}}{T} \nabla T\right)
$$

where $x$ is the fractional concentration of solute (i.e., helium) and $k_{T}$ is the Soret (or thermodiffusion) coefficient. This coefficient is not small in general: it can be as large as of order unity, and can have either sign. In a metal, an apparently successful model for $k_{T}$ (Bhat and Swalin 1971) evaluates this coefficient as the sum of a "dense gas" contribution (determined by the mass and size of the pseudoatoms) and an electronic contribution, given by Gerl (1967). The 
former was evaluated using the hard sphere diameters implied by thermodynamics, and the latter was evaluated using the conductivity calculations of Stevenson and Ashcroft (1974). Both contributions were positive and approximately $0.5 x$ each, where $x$ is the (assumed small) helium number fraction. In the situations of interest, we might therefore expect $k_{T} \approx+0.1$. As in the case of molecular diffusion, this result should be viewed with suspicion if the fluid is near a phase transition. A positive value of $k_{T}$ implies that the helium tends to diffuse toward colder regions. In most of the considerations in Paper II, $k_{T}$ should be small enough to only slightly modify the solute flux (and certainly not change the direction of flux). We shall therefore ignore it.

\section{TRANSPORT PROPERTIES OF THE MOLECULAR PHASE}

We repeat the considerations of the last section, but for the molecular phase.

\section{a) Electrical Conductivity}

Except near the molecular-metallic phase transition, molecular hydrogen is an insulator, and the only electrical conduction arises from impurities (Smoluchowski 1972). However, quite general considerations, together with recent band-structure calculations (Friedli and Ashcroft 1976), indicate that the indirect band gap in molecular hydrogen vanishes at or near the molecular-metallic transition. Smoluchowski (1975) has pointed out that under these circumstances, the electronic conductivity at the phase transition could be within an order of magnitude of that given by equation (10).

\section{b) Thermal Conductivity}

If electrical conduction is almost metallic at the phase transition, then heat can be transported by electrons, with $\kappa \approx 0.1 \mathrm{~cm}^{2} \mathrm{~s}^{-1}$ (eq. [12]). If no electronic degrees of freedom are available, then the less efficient molecular motions must be utilized. Neglecting the internal motion of the hydrogen molecule, this implies

$$
\kappa \approx c \sigma\left(\frac{k_{B} T}{M}\right)^{1 / 2},
$$

where $c$ is a correction factor of order unity, $\sigma$ is a hard sphere diameter, and $M$ is the mass of the molecule. The correction factor can be deduced from Chapman-Enskog theory, or from Monte Carlo results for hard spheres (Alder, Gass, and Wainwright 1970). As usual, the hard sphere diameter is deduced from thermodynamic models (e.g., § IV). For a hydrogen-rich fluid, the molecular contribution to $\kappa$ is then

$$
\kappa \approx 10^{-2} T_{4}^{-1 / 2} \mathrm{~cm}^{2} \mathrm{~s}^{-1},
$$

accurate to perhaps a factor of 2 , for $\rho \approx 1 \mathrm{~g} \mathrm{~cm}^{-3}$.

\section{c) Viscosity}

Dense molecular fluids, like gases, have a Prandtl number close to unity. This property is predicted by kinetic theories and Monte Carlo calculations (Alder, Gass, and Wainwright 1970), which show that both viscosity and thermal conductivity vary linearly as the Enskog correction. We shall not attempt to evaluate the Prandtl number more accurately, so it is adequate to use

$$
\nu \approx 10^{-2} T_{4}^{-1 / 2} \mathrm{~cm}^{2} \mathrm{~s}^{-1} .
$$

If electronic transport is negligible, then $\operatorname{Pr} \approx 1$. If electronic transport is almost metallic, then $\mathrm{Pr} \approx 0.1$ or even 0.01 .

\section{d) Self-Diffusion}

This transport coefficient is comparable to $\nu$, but varies inversely as the Enskog correction and thus has a different density and temperature dependence. Using equation (21), with $c$ given by Monte Carlo results (Alder, Gass, and Wainwright 1970), one finds

$$
D \approx 4 \times 10^{-3} \rho^{-5 / 6} T_{4}^{3 / 2} \mathrm{~cm}^{2} \mathrm{~s}^{-1}
$$

for pure hydrogen or pure helium, to within a factor of 2 .

\section{e) Interdiffusion}

The thermodynamic calculations ( $($ IV) indicate that the $\mathrm{H}_{2}$ molecule is $15 \%$ larger than the helium atom. The diffusion of a small amount of helium in hydrogen should therefore proceed slightly faster than the selfdiffusion of hydrogen. This effect is smaller than the probable inaccuracies in the calculation, so equation (24) suffices for the interdiffusion. As in the metallic case, this result should be viewed with caution near phase transitions.

\section{f) Second-Order Transport Coefficients}

The only second-order coefficient that is likely to be important is $k_{T}$, the Soret coefficient. The dense-gas theory (Chapman and Cowling 1952) predicts $k_{T} \approx$ $0.5 x$, where $x$ is the (assumed small) helium mole fraction. The positive value is ensured by the greater mass of the helium atom and the strongly repulsive character of the intermolecular potentials. As usual, this result is suspect near phase transitions.

\section{g) Radiative Opacity}

Unlike the preceding discussion, which has concentrated on the dense fluid regime $(\rho \approx 0.1$ to $1 \mathrm{~g}$ $\mathrm{cm}^{-3}$ ), the radiative opacity is of interest for a much wider range of densities and temperatures. Interior models of Jupiter, for example, always assume an adiabatic molecular envelope, and do not allow for the possibility that molecular hydrogen may be sufficiently transparent for radiation to transport the internal heat flux subadiabatically. Stevenson (1976a) has considered this problem, and concludes that molecular hydrogen 
alone is sufficiently opaque to ensure convection, except at temperatures and pressures for which the $1500 \mathrm{~cm}^{-1}$ to $3000 \mathrm{~cm}^{-1}$ window in the hydrogen spectrum is important. These calculations are based on the theory and observations of Linsky (1969), Welsh (1969), and Herzberg (1952). In Jupiter, the $1500 \mathrm{~cm}^{-1}$ to $3000 \mathrm{~cm}^{-1}$ window is most important for $400 \mathrm{~K} \leqslant T \leqslant 700 \mathrm{~K}$. For $T \leqslant 400 \mathrm{~K}$, pure translational and rotation-translational pressure-induced bands provide sufficient opacity to ensure convection, until the optical depth to free space becomes less than unity at $T \approx 150 \mathrm{~K}$ (Trafton and Stone 1974; Wallace, Prather, and Belton 1974). At $T \geqslant 700 \mathrm{~K}$, the vibration-rotation translational band $(\nu \approx 4000$ $\left.\mathrm{cm}^{-1}\right)$, and higher-order bands $\left(\nu \approx 8000 \mathrm{~cm}^{-1}\right.$, $12,000 \mathrm{~cm}^{-1}$ ) ensure convection in Jupiter. Since the pressure-induced opacity varies roughly as $P^{2}$, where $P$ is the pressure, and since the bands become broadened and overlapping at higher pressures, the radiative heat transport decreases as one goes deeper into the planet. At even higher temperatures $(T \geqslant$ $3000 \mathrm{~K}$ ) free-free absorption, arising from the small number of conduction electrons in the molecular fluid, begins to dominate. Unlike the free-free absorption usually considered (e.g., Clayton 1968), the molecular fluid is so dense that the electron-molecule interactions are more important than electron-ion interactions in ensuring momentum conservation.

The region $400 \mathrm{~K} \leqslant T \leqslant 700 \mathrm{~K}$ is nevertheless probably convective, but only because of the small amounts of strongly absorbing molecules such as $\mathrm{H}_{2} \mathrm{O}, \mathrm{CH}_{4}$, and $\mathrm{NH}_{3}$. The opacities of these species are "spiky" at room temperature, with typical strong line separations of about $1 \mathrm{~cm}^{-1}$. However, the pressure broadening exceeds the line spacing for pressures in excess of 5 or 10 bars, so that the opacity becomes quasi-continuous. Assuming the validity of the quasicontinuous approximation, Stevenson (1976a) estimates that $\mathrm{H}_{2} \mathrm{O}, \mathrm{CH}_{4}$, and $\mathrm{NH}_{3}$ have sufficient combined opacity to "block" the $1500 \mathrm{~cm}^{-1}$ to 3000 $\mathrm{cm}^{-1}$ hydrogen window in Jupiter. The data used in this calculation were Ferriso, Ludwig, and Thomson (1966) for $\mathrm{H}_{2} \mathrm{O}$; Burch and Williams (1962) and Plyler, Tidwell, and Blaine (1960) for $\mathrm{CH}_{4}$; and Gille and Lee (1969) and Benedict, Plyler, and Tidwell (1958) for $\mathrm{NH}_{3}$. Some uncertainty does remain, however, especially in the $2000-2500 \mathrm{~cm}^{-1}$ region where none of $\mathrm{H}_{2} \mathrm{O}, \mathrm{CH}_{4}$, or $\mathrm{NH}_{3}$ is strongly absorbing, so a careful band model is probably desirable.

To conclude: A hydrogen-helium mixture is not sufficiently opaque to ensure convection in the deep atmosphere under typical conditions (such as those which prevail in Jupiter). The addition of a solar abundance of minor constituents $\left(\mathrm{H}_{2} \mathrm{O}, \mathrm{CH}_{4}, \mathrm{NH}_{3}\right)$ probably suffices to reduce the radiative heat transport to less than $10 \%$ of the total and ensure an adiabatic thermal structure.

\section{CONCLUSION}

It is evident from our discussion of the phase diagram that the main uncertainty lies in the value of the critical temperature for the pure molecularmetallic hydrogen transition. Whereas this critical value is only known to about an order of magnitude, the metallic $\mathrm{H}-\mathrm{He}$ critical temperature is known to perhaps $20 \%$, and the $\mathrm{H}_{2}$-He critical temperature to perhaps a factor of 2 . This uncertainty forces us to consider a wide range of possibilities in Paper II (Stevenson and Salpeter 1977), where specific thermal and compositional evolutions are discussed. Improvements in the value of the molecular-metallic hydrogen critical temperature will not be easy from purely theoretical calculations, and some experimental input is highly desirable.

The partitioning of minor constituents is clearly difficult to predict quantitatively, with the exception of deuterium. It is particularly desirable to understand more about the high-pressure properties of $\mathrm{H}_{2} \mathrm{O}$. Generally speaking, the relevant temperature $\left(\sim 10^{4}\right.$ $\mathrm{K}$ ) is too great for highly nonuniform partitioning of the kind that is observed in the Earth, for example. Constituents such as $\mathrm{H}_{2} \mathrm{O}, \mathrm{CH}_{4}$, and $\mathrm{NH}_{3}$ probably prefer molecular or helium-rich phases.

With two notable exceptions (electronic conductivity and radiative opacity of the molecular phase), the transport properties are known to within a factor of 3 , typically. This is usually quite adequate for the purposes of Paper II. The uncertainty in the electronic conductivity of the molecular phase near the molecular-metallic phase transition is of concern, since if electronic degrees of freedom are available for heat transport, then the efficiency of upward transport of helium by convection is generally low (see Paper II). The uncertainty in the radiative opacity is generally only large at those temperatures and pressures for which the opacity is one or more orders of magnitude in excess of that required to transport the heat flux at an adiabatic temperature gradient.

Apart from the radiative opacity, where minor constituents are crucial, the effect of such molecules as $\mathrm{H}_{2} \mathrm{O}, \mathrm{CH}_{4}$, and $\mathrm{NH}_{3}$ on the phase diagram and transport properties is small, provided their abundances are close to solar.

We wish to thank N. W. Ashcroft, M. E. Fisher, W. B. Hubbard, and R. Smoluchowski for discussions and comments. This work is supported by National Aeronautics and Space Administration grant NGR 33-010-188 and National Science Foundation grant AST 75-21153.

\section{REFERENCES}

Abraham, F. R. 1975a, J. Chem. Phys., 63, 157. 1975b, J. Chem. Phys., 63, 1316.

Abraham, F. R., Schreiber, D. E., Mruzik, M. R., and Pound,

G. M. 1976, Phys. Rev. Letters, 36, 261.
Abrikosov, A. A. 1964, Soviet Astr.-AJ, 31, 112

Alder, B. J., Gass, B. M., and Wainwright, T. E. 1970, J. Chem. Phys., 53, 3813.

Ascarelli, P., and Paskin, A. 1968, Phys. Rev., 165, 222. 
Ashcroft, N. W. 1968, Phys. Rev. Letters, 21, 1748.

Ashcroft, N. W., and Langreth, D. C. 1967, Phys. Rev., $155,682$.

Aviram, I., Goshem, S., Rosenfeld, Y., and Thieberger, R. 1976, J. Chem. Phys., 65, 846.

Baym, G. 1964, Phys. Rev., A135, 1691.

Beer, R., Farmer, C. B., Norton, R. H., Martonchik, J. V., and Barns, T. G. 1972, Science, 175, 1360.

Benedict, W. S., Plyler, E. A., and Tidwell, E. D. 1958, J. Chem. Phys., 29, 829.

Bhat, B. N., and Swalin, R. A. 1971, in Atomic Transport in Solids and Liquids, ed. A. Lodding and T. Lagerwall (Tübingen: Tübingen-Verlag), p. 179.

Brovman, E. G., Kagan, Y., and Kholas, A. 1972, Soviet Phys.-JETP, 35, 783.

Brust, D. 1972, Phys. Letters, 40A, 255.

Burch, D. E., and Williams, D. 1962, Appl. Opt., 1, 587.

Caldwell, D. R. 1973, J. Phys. Chem., 77, 2004.

Caron, L. G. 1974, Phys. Rev., 9B, 5025.

Chapman, S., and Cowling, T. G. 1952, The Mathematical Theory of Non-Uniform Gases (Cambridge: Cambridge University Press), p. 254.

Clayton, D. D. 1968, Principles of Stellar Evolution and Nucleosynthesis (McGraw-Hill), chap. 3.

Dyson, F. 1971, Ann. Phys., 63, 1 .

Ejima, T., and Yamamura, T. 1973, in The Properties of Liquid Metals, ed. S. Takenchi (London: Taylor and Francis), p. 537.

Etters, R. D., Danilowicz, R., and England, W. 1975, Phys. Rev., 12A, 2199.

Ferriso, C. C., Ludwig, C. B., and Thomson, A. L. 1966, J. Quant. Spectrosc. Rad. Transf., 6, 241.

Filinov, V. S., and Norman, E. G. 1975, Phys. Letters, 55A, 219.

Firey, B., and Ashcroft, N. W. 1976, unpublished.

Friedli, C., and Ashcroft, N. W. 1976, unpublished.

Gerl, M. 1967, J. Phys. Chem. Solids, 28, 725.

Gille, J. C., and Lee, T-H. 1969, J. Atm. Sci., 26, 932.

Glyde, H. R., Keech, G. H., Mazighi, R., and Hansen, J. P. 1976, Phys. Letters, 58A, 226.

Grigoryev, F. V., Kormer, S. B., Mikhailova, O. L., Tolochko, A. P., and Urlin, V. D. 1972, JETP Letters, 16, 201.

Hamann, S. D., and Linton, M. 1966, Trans. Faraday Soc., $62,2234$.

Hammerberg, J., and Ashcroft, N. W. 1974, Phys. Rev., 9B, 409.

Hansen, J. P., McDonald, I. R., and Pollock, E. L. 1975, Phys. Rev., A11, 1025.

Hansen, J. P., and Vieillefosse, P. 1976, Phys. Rev. Letters, 37, 391.

Herzberg, G. 1952, Ap. J., 115, 337.

Hubbard, W. B. 1974, Ap. J., 190, 223.

Hubbard, W. B., and DeWitt, H. 1976, unpublished.

Hubbard, W. B., and Lampe, M. 1969, Ap. J. Suppl., 18, 297.

Hubbard, W. B., and Slattery, W. L. 1971, Ap. J., 168, 131. . 1976, in Jupiter, ed. T. Gehrels (University of Arizona Press), p. 176.

Hubbard, W. B., and Smoluchowski, R. 1973, Space Sci. Rev., 14, 599.

Kerley, G. I. 1972, Phys. Earth Planet. Inter., 6, 78.

Krumhansl, J., and Wu, S-W. 1968, Phys. Letters, 28A, 263.

Landau, L., and Lifshitz, E. M. 1959, Fluid Mechanics (Reading, Massachusetts: Addison-Wesley), p. 224.

Landau, L., and Zel'dovich, G. 1943, Acta Phys. Chim. (USSR), 18, 194

Larson, H. P., Fink, U., Treffers, R. R., and Gautier, T. N. 1975, Ap. J. (Letters), 197, L137.

Lebowitz, J., and Rowlinson, J. S. 1964, J. Chem. Phys., 41, 133 .

Linsky, J. L. 1969, Ap. J., 156, 989.
Longuet-Higgins, H. C., and Pople, J. A. 1956, J. Chem. Phys., 25, 884 .

McMahan, A., Beck, H., and Krumhansl, J. 1974, Phys. Rev., 9A, 1852.

Monkhurst, H. J., and Oddershede, J. 1973, Phys. Rev. Letters, 30, 797.

Neece, G. A., Rogers, F. J., and Hoover, W. G. 1971, J. Comput. Phys., 7, 621.

Østgaard, E. 1974, Physica, 74, 113.

Plyler, E. A., Tidwell, E. D., and Blaine, L. R. 1960, J. Res. $N B S, 64 \mathrm{~A}, 201$.

Podolak, M. 1977, Icarus, 30, 155.

Podolak, M., and Cameron, A. G. W. 1975, Icarus, 25, 627.

Pollock, E. L., and Alder, B. 1977, UCRL Rept. No. 79511.

Pollock, E. L., and Hansen, J. P. 1973, Phys. Rev., 8A, 3110.

Ramaker, D. E., Kumar, L., and Harris, F. E. 1975, Phys. Rev. Letters, 34, 812.

Ramsey, W. H. 1963, M.N.R.A.S., 125, 469.

Ree, F. H., and Bender, C. F. 1974, Phys. Rev. Letters, 32, 85.

Ross, M. 1972, J. Chem. Phys., 56, 4651. 1974, J. Chem. Phys., 60, 3634.

Ross, M., and McMahan, A. L. 1976, Phys. Rev., B13, 5154.

Salpeter, E. E. 1954, Australian J. Phys., 7, 353.

Salpeter, E. E., and Zapolsky, H. 1967, Phys. Rev., 158, 876.

Shafer, R., and Gordon, R. G. 1973, J. Chem. Phys., 58, 5422.

Silver, D. M., and Stevens, R. M. 1973, J. Chem. Phys., 59, 3378.

Simcox, L. N., and March, N. H. 1962, Proc. Phys. Soc. London, 80, 830.

Smoluchowski, R. 1971, Ap. J., 166, 435.

. 1972, Phys. Earth Planet. Inter., 6, 48

- 1973, Ap. J. (Letters), 185, L95.

1975, Ap. J. (Letters), 200, L119.

Stevenson, D. J. 1975, Phys. Rev., 12B, 3999. $1976 a$, Ph.D. thesis, Cornell University. 1976b, Phys. Letters, 58A, 282.

Stevenson, D. J., and Ashcroft, N. W. 1974, Phys. Rev., 9A, 782.

Stevenson, D. J., and Salpeter, E. E. 1976, in Jupiter, ed. T. Gehrels (University of Arizona Press), p. 85.

. 1977, Ap. J. Suppl., 35, 239 (Paper II).

Straus, D. M., and Ashcroft, N. W. 1977, Phys. Rev. Letters, 38, 415 .

Straus, D. M., Ashcroft, N. W., and Beck, H. 1977, in preparation.

Streett, W. B. 1976, Ap. J., 186, 1107.

Stroud, D. 1973, Phys. Rev., B7, 4405

Stroud, D., and Ashcroft, N. W. 1972, Phys. Rev., B5, 371.

Trafton, L. M., and Stone, P. H. 1974, Ap. J., 188, 649.

Trauger, J. T., Roesler, F. L., Carleton, N. P., and Traub, W. A. 1973, Ap. J. (Letters), 184, L137.

Trubitsyn, V. P. 1967, Soviet Phys.-Solid State, 8, 2593.

. 1972, Soviet Astr.-AJ, 16, 342.

Vadovic, C. J., and Colver, C. P. 1971, Phil. Mag., 24, 509.

Van Horn, H. 1967, Phys. Rev., 157, 342.

Vereschchagin, L. F., Yakovlev, E. N., and Timofeev, Yu. A. 1975a, JETP Letters, 21, 85.

- 1975b, JETP Letters, 21, 304.

Vieillefosse, P., and Hansen, J. P. 1975, Phys. Rev., A12, 1106.

Wainwright, T., and Alder, B. 1958, Il Nov. Cimento Suppl., p. 116.

Wallace, L., Prather, M., and Belton, M. J. S. 1974, Ap. J., 193, 481 .

Welsh, H. L. 1969, J. Atm. Sci., 26, 835.

Wigner, E., and Huntington, H. B. 1935, J. Chem. Phys., 3, 764.

Witten, T. A., Jr. 1974, Ap. J., 188, 615.

Zharkov, V. N., Makalkin, A. B., and Trubitsyn, V. P. 1975 , Soviet Astr.-AJ, 18, 768. 